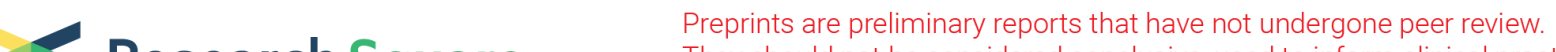

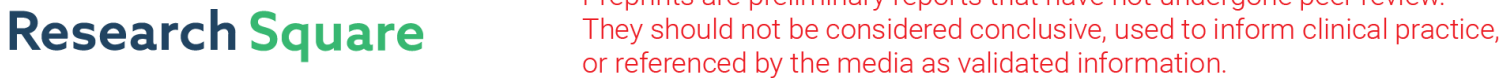

\section{m6A-mediated upregulation of LINC01578 promotes the progression of glioma by modulating the miR-6893-3p/TRIM14 axis}

\section{Xinwei Wu}

The First Affiliated Hospital of Wannan Medical College: Yijishan Hospital of Wannan Medical College Hui Yang

The First Affiliated Hospital of Wannan Medical College: Yijishan Hospital of Wannan Medical College Xiaolong Zhu

The First Affiliated Hospital of Wannan Medical College: Yijishan Hospital of Wannan Medical College

Min Zhong

The First Affiliated Hospital of Wannan Medical College: Yijishan Hospital of Wannan Medical College

\section{Mengying Zhang}

The First Affiliated Hospital of Wannan Medical College: Yijishan Hospital of Wannan Medical College

Kun Lv ( Ivkun315@126.com )

The First Affiliated Hospital of Wannan Medical College: Yijishan Hospital of Wannan Medical College

\section{Research Article}

Keywords: LINC01578, Glioma, miR-6893-3p, TRIM14, N6-methyladenosine

Posted Date: January 28th, 2022

DOI: https://doi.org/10.21203/rs.3.rs-1285353/v1

License: (c) (1) This work is licensed under a Creative Commons Attribution 4.0 International License. Read Full License 


\section{Abstract \\ Background}

Long noncoding RNAs (IncRNAs) play crucial roles in tumor progression and are dysregulated in glioma. However, the functional roles of IncRNAs in glioma remain largely unknown.

\section{Methods}

Using the TCGA and GEPIA2 databases, the overexpression of LINC01578 in glioma tissues was determined and further validated in glioma cell lines. The effects of LINC01578 on proliferation, migration, and invasion in glioma were detected by in vitro and in vivo experiments. RNA immunoprecipitation (RIP), dual luciferase reporter, RNA FISH, and RNA sequencing assays were carried out to elucidate the underlying molecular mechanisms regulated by LINC01578 in glioma. RIP and RTqPCR was used to analyze the regulatory effect of $N^{6}$-methyladenosine $\left(m^{6} \mathrm{~A}\right)$ on aberrantly expressed LINC01578.

\section{Results}

Highly expressed LINC01578 was identified in glioma tissues and was associated with poor prognosis in glioma patients. Functional assays showed that LINC01578 regulates glioma growth and metastasis in vitro and in vivo. Mechanistically, LINC01578 sponged miR-6893-3p to upregulate TRIM14 expression, thereby facilitating glioma progression. Furthermore, LINC01578 was upregulated in response to $N^{6}$ methyladenosine modification, which was attributed to METTL3/YTHDF1-mediated RNA transcripts.

\section{Conclusion}

Our results reveal a novel $\mathrm{m}^{6} \mathrm{~A} / \mathrm{LINC01578/miR-6893-3p/TRIM14} \mathrm{pathway} \mathrm{for} \mathrm{glioma} \mathrm{progression} \mathrm{and}$ suggest LINC01578 as a novel prognostic indicator and therapeutic target for glioma.

\section{Background}

Glioma is the most aggressive and fatal central nervous system tumor due to the high infiltration and invasion rate of glioma cells [1]. Glioma are classified by the World Health Organization (WHO) into grades I - IV, based on malignant behavior [2]. The highly malignant brain tumor glioblastoma (GBM) has a dismal median patient survival of $\sim 15$ months, despite the current aggressive standard therapy of surgical resection followed by radiotherapy and temozolomide (TMZ) chemotherapy [3]. The mechanism of this type of tumorigenesis is currently unclear, and it often infiltrates into normal brain tissue [4]. 
Therefore, exploration of the mechanism behind the occurrence and development of gliomas and identification of potential biomarkers for timely intervention is crucial.

Recently, the role of non-coding RNAs (ncRNAs) in cancer has attracted increasing attention [5]. Long non-coding RNAs (IncRNAs), defined as ncRNAs more than 200 nucleotides in length, have been reported to be dysregulated in many human cancer types [6]. LncRNAs exert their functions at the translational, transcriptional, and posttranscriptional level and have been found to be involved in cell survival, cell growth, and differentiation [7]. Moreover, it is interesting to note that IncRNAs may play an important role in angiogenesis in glioma [8]. Long noncoding RNA LINC01578 (Chaserr) is a conserved IncRNA located upstream of Chd2 that regulates CHD2 expression [9]. LINC01578 was upregulated in colon cancer and drives colon cancer metastasis through a positive feedback loop via the NF-KB/YY1 axis [10]. However, the roles of LINC01578 in glioma metastasis are still largely unclear.

The $N^{6}$-methyladenosine $\left(\mathrm{m}^{6} \mathrm{~A}\right) \mathrm{RNA}$ modification, the most abundant internal epi-transcriptomic modification, is found in miRNAs, IncRNAs, and circRNAs [11]. $\mathrm{m}^{6} \mathrm{~A}$ methylation is modified by a protein complex consisting of methyltransferases (writers) such as METTL3, METTL14, and Wilm's tumor protein (WTAP), and is likely to occur in the RRACH motif (R; A or G, H; except for G). This modification is reversible since demethylases such as FTO and ALKBH5 have also been discovered. The $\mathrm{m}^{6} \mathrm{~A}$ modification may require readers, such as the YTH domain-containing 1 (YTHDC1), YTHDC2, YTH $N^{6}$ methyladenosine RNA-binding protein 1 (YTHDF1), YTHDF2, and YTHDF3 [12-14]. These readers regulate RNA processing or metabolism including mRNA alternative splicing, export, translation, and decay [15]. Increasing numbers of studies have shown that $\mathrm{m}^{6} \mathrm{~A}$ modification and its associated regulatory proteins play pivotal roles in the pathogenesis of glioma [16-18]. However, the relationship between the oncogenic role of LINC01578 and $\mathrm{m}^{6} \mathrm{~A}$ modification remains unclear.

In the current study, we determined LINC01578 expression in glioma tissues and revealed its association with the overall survival of glioma patients. We also analyzed the contribution of LINC01578 to the function of glioma in vitro/vivo and the underlying mechanism. We also examined the relationship between dysregulated LINC01578 and $\mathrm{m}^{6} \mathrm{~A}$ modification. Our findings clarify the role of the LINC01578/miR-6893-3p/TRIM14 axis in the tumorigenesis of glioma and suggest LINC01578 as a potential diagnostic and prognostic marker.

\section{Materials And Methods}

\section{Patients samples}

Normal human brain and glioma tissues were removed during surgery in the Department of Neurosurgery, the first affiliated Hospital of Wannan Medical College. Tissue samples were snap-frozen in liquid nitrogen and stored at $-80^{\circ} \mathrm{C}$ for RNA extraction. Informed consent was obtained from all participants and/or their legal guardians. This study was approved by the Ethics Committee of the first affiliated Hospital of Wannan Medical College. 


\section{Cell lines and cell culture}

Four human glioma cell lines (U373, U118, A172, T98G), one normal human glial cell line (HEB), and normal human renal epithelial cells (293T) were purchased from American Type Culture Collection (ATCC, Manassas, VA). All cells were cultured in Dulbecco's modified eagle medium (DMEM) (Hyclone, GE Healthcare Life Sciences, Logan, UT, USA) and grown in an incubator at $37^{\circ} \mathrm{C}$ and $5 \% \mathrm{CO}_{2}$ in DMEM mixed with $10 \%$ (v/v) fetal bovine serum (FBS) (Gibco, Life Technologies, Grand Island, NY).

\section{RNA extraction and RT-qPCR}

Total RNA from frozen brain tissues and cultured cells were extracted using Trizol reagent (Ambion, life technologies, Carlsbad, CA, USA) as previously described [19]. cDNA from total RNA was synthesized using RevertAid First Strand cDNA Synthesis Kit (Thermo Scientific, Vilnius, Lithuania, USA). The gene expression were measured by real-time quantitative PCR utilizing QuantiNova ${ }^{\text {TM }}$ SYBR ${ }^{\circledR}$ Green PCR Kit (Qiagen, Hilden, Germany) on the CXF96 PCR system (Bio-Rad, Hercules, CA, USA). The primer sequences were as follows in Table S1. GAPDH and U6 were served as internal control.

\section{Cell transfection}

The short hairpin RNAs (shRNA) lentivirus (sh-NC, sh-LINC01578) was purchased from HANBIO (Shanghai, China). Lentiviruses were transfected into glioma cells for $48 \mathrm{~h}$, and stable cell clones were selected after one week using puromycin $(5 \mu \mathrm{g} / \mathrm{ml})$. Small interfering RNA (siRNA), microRNA mimics (agomir), and microRNA inhibitor (antagomir) were purchased from RiboBio (Guangzhou, China) and transfected using the riboFECT ${ }^{\mathrm{TM}} \mathrm{CP}$ Transfection Kit (RiboBio) via the manufacturer's protocol. For overexpression, the LINC01578 cDNA was cloned into the pTSB02-GFP-PURO vector and transfected using Lipofectamine 3000 (Invitrogen, Carlsbad, CA) following the manufacturer's instructions. For YTHDF1 RNA interference, the cells were transfected with $30 \mathrm{nM}$ siRNA (GenePharma, China) targeting the YTHDF1 and a control siRNA using Lipofectamine RNAiMAX (ThermoFisher, 3778150, USA) following the manufacturer's instructions. The sequences used are provided in Table S2.

\section{Cell proliferation and colony formation}

Cell proliferation was determined using a Cell-Light EdU Apollo567 In Vitro Kit (RiboBio; Guangzhou; China), following the manufacturer's instructions. Finally, the cells were captured using a fluorescence microscopy. For the colony formation assay, glioma cells were seeded in 6-well plate at 5000 cells/well. Approximately 2 weeks later, the cells were fixed in paraformaldehyde and stained with crystal violet. The colonies were imaged and counted.

\section{Invasion and migration assays}

Transwell chambers were used for the migration assays. Cells $\left(2 \times 10^{4}\right)$ cells were suspended in $100 \mu \mathrm{l}$ serum-free medium and seeded in the upper chamber. For invasion assays, the upper chambers were precoated with diluted Matrigel (356234; BD Biosciences) and $4 \times 10^{4}$ cells were added to the upper 
chamber. The lower chamber contained $600 \mu \mathrm{L}$ DMEM medium with $10 \%$ serum. After $24 \mathrm{~h}$, the migrated or invasive cells were fixed, stained, and counted.

Wound-healing assay was also used for the migration assay. The glioma cells were seeded on 6-well cell culture plates and scratched using $200 \mu$ pipettes. The cells were cultured in DMEM medium without FBS and a microscope was used to capture photographs at $24 \mathrm{~h}$.

\section{Fluorescence in situ hybridization(FISH)}

Specific FISH probes for LINC01578 were designed and synthesized by RiboBio (Guangzhou, China) and miR-6893-3p was designed and synthesized by Boster (Wuhan, China). Glioma cells grown on glass coverslips were fixed in $4 \%$ paraformaldehyde (PFA) for 30 minutes and permeabilized with $0.5 \%$ Triton X100. Then, cells were washed three times with phosphate buffer saline (PBS) and treated with prehybridization buffer ( $2 \times$ saline sodium citrate, $10 \%$ formamide). The FISH probes for LINC01578 were resuspended in the hybridization buffer ( $2 \times$ saline sodium citrate, $10 \%$ formamide, $10 \%$ dextran sulphate) to a final concentration of $250 \mathrm{nmol} / \mathrm{L}$ per probe set. Hybridization was carried out in a humidified chamber at $37^{\circ} \mathrm{C}$ for 16 hours, and then cells were washed three times with PBS. Then, the FISH probes for miR-6893-3p were resuspended in the hybridization buffer, and hybridization was carried out in a humidified chamber at $37^{\circ} \mathrm{C}$ for 16 hours. After incubation, cells were washed three times with $4 \times$ saline sodium citrate (SSC) plus with $0.1 \%$ Tween -20 at $42^{\circ} \mathrm{C}$, followed by one wash with $2 \times \mathrm{SSC}$ and one wash with $1 \times \mathrm{SSC}$ at $42^{\circ} \mathrm{C}$. Cells were then washed three times with PBS and nuclei were labelled with DAPI. We continued with several rounds of washing and finished with mounting the coverslip onto a microscope slide using an anti-fade mounting medium. For the fluorescence in situ hybridization of glioma tissues, the tissues were fixed with neutral formalin and the paraffin-embedded tissue sections were placed on clean glass slides. The slides were placed on a baking sheet machine at $65^{\circ} \mathrm{C}$ overnight. Tissue sections were then soaked in a dye vat filled with xylene for 10 minutes and then immediately immersed in $100 \%$ ethanol, $85 \%$ ethanol, and $70 \%$ ethanol for 2 minutes followed by immersion in deionized water for 3 minutes. After the sections were removed, they were dried with a lint-free paper towel to remove excess water, immersed in $2 \times \mathrm{SSC}$ solution, and rinsed for 5 minutes. Sections were then treated with proteinase $\mathrm{K}$ working solution at $37^{\circ} \mathrm{C}$ for 30 minutes, immersed in $2 \times \mathrm{SSC}$ solution for 5 minutes, and rinsed sequentially in $70 \%$ ethanol, $85 \%$ ethanol, and $100 \%$ ethanol for 2 minutes and then dried naturally. After mixing the LINC01578/miR-6893-3p, $10 \mu$ l drops were deposited on the hybridization area of the tissue section and covered with a cover glass. Sections were then placed on the hybridization instrument, denatured at $83^{\circ} \mathrm{C}$ for $5 \mathrm{~min}$, and hybridized at $42^{\circ} \mathrm{C}$ for $16 \mathrm{~h}$. Then, the cover glass was removed and the hybridized sections were placed in $0.4 \times$ SSC solution and $2 \times$ SSC solution for 2 minutes in turn, soaked in $70 \%$ ethanol for 3 minutes, and dried naturally. Finally, $10 \mu$ of DAPI counterstaining solution was dropped on the hybridization area, covered with a cover glass, and hybridized for 20 minutes. All images were analyzed on a confocal laser scanning microscope (Zeiss LSM800 confocal microscope, Mainz, Germany). The FISH probe sequence was as follows: miR-6893-3p: 5'-CTGGCAGGTGAAGGCAGCAGGG -3'. 


\section{RNA immunoprecipitation(RIP)}

RIP assay was performed using a Magna RIP RNA-binding Protein Immunoprecipitation Kit (Millipore, MA, USA) in accordance with the manufacturer's protocol. Glioma cells were isolated and lysed by RIP lysis buffer and incubated with antibodies against AGO2 (Abcam, Cambridge, UK), or m6A (Abcam, Cambridge, UK) at $4{ }^{\circ} \mathrm{C}$ overnight. IgG was used as negative control. The immunoprecipitated RNAs were eluted and analyzed by RT-qPCR.

\section{Western blotting(WB)}

TRIM14 was purchased from Proteintech (Chicago, USA). PTEN, p-Akt, Akt, p-mTOR, mTOR, p-P70S6K, P70S6K, Cyclin D1, Cyclin E1, PCNA were purchased from Cell Signaling Technology (Beverly, MA, USA). E-cadherin, N-cadherin, MMP-9 and MMP-2 antibodies were obtained from Santa Cruz Biotechnology (Dallas, TX, USA). HRP-conjugated and anti-rabbit IgG secondary antibodies were obtained from Cell Signaling Technology (Beverly, MA, USA). Cells or tissues were harvested the indicated times and lysed in radio immunoprecipitation assay (RIPA) extraction solution. The protein concentration was determined by a BCA Protein Assay kit (Beyotime, China). Total protein lysates ( $25 \mathrm{mg} / \mathrm{lane}$ ) were subjected to SDSPAGE and transferred onto an Immobilon polyvinylidene difluoride (PVDF) membrane (Millipore, MA, USA). The membranes were blocked for $1 \mathrm{~h}$ with $5 \%$ non-fat dry milk at room temperature and were incubated with diluted primary antibodies at $4^{\circ} \mathrm{C}$ overnight, followed by treatment with HRP-conjugated secondary antibodies for $1 \mathrm{~h}$ at room temperature. Antibody-antigen complexes were visualised using either a LI-COR Odyssey Infrared Imaging System (LI-COR Biosciences) or a chemiluminescence imaging system (Clinx, Shanghai, China). Protein levels were semi-quantified using Image J 1.52 software.

\section{Luciferase activity experiment}

The 293T and A172 cells $\left(1 \times 10^{5}\right.$ cells/well) were seeded into 24 -well plates. Cell were co-transfected with the constructed luciferase plasmids and miR-6893-3p mimics or negative control using Lipofectamine 3000 reagent (Invitrogen, USA) for $48 \mathrm{~h}$. Then, firefly and renilla luciferase activities were measured using the Dual-Luciferase Reporter Assay System (Promega, Madison, WI, USA) following the manufacturer's protocol.

\section{In vivo tumor xenotranplantation}

Xenograft athymic mice models were constructed to examine the effects of LINC01578 on glioma tumor growth in vivo. BALB/C athymic mice are fed in an SPF environment (temperature: $23-24^{\circ} \mathrm{C}$, humidity: 30 $50 \%$ ), and free food and water are provided throughout the process. Glioma cells with shLINC01578 or shNC and corresponding control cells were injected subcutaneously into the flanks of 4-week-old BALB/c athymic mice (weighting approximately $20 \mathrm{~g}$ each $₫ 5 \times 10^{6}$ cells resuspended in $200 \mathrm{~mL}$ of culture medium). Tumors were measured routinely with a caliper. The xenograft volume was calculated as (longest diameter $\times$ smallest diameter ${ }^{2}$ ) $/ 2$. 
$\mathrm{BALB} / \mathrm{c}$ athymic mice were sacrificed 30 days later and tumors were collected. During the experiment, observe at least three times a week. The clinical symptoms of the experimental animals were evaluated and consulted with professional veterinarians and the health and welfare of the animals were put first. For animals in pain, analgesics were used to relieve them in time. All animals were euthanized by intravenous injection of $100 \mathrm{mg} / \mathrm{kg}$ sodium pentobarbital. the xenografts were harvested and weighed. All animal experiments were approved by the Animal Ethics Committee of the First Affiliated Hospital of Wannan Medical College.

\section{Bioinformatics analysis}

Differentially expressed genes data analyses and survival analysis. We used including the Cancer Genome Atlas database (TCGA. http://tcga-data.nci.nih.gov/tcga/), the Gene Expression Profiling Interactive Analysis 2 databases囚GEPIA2. http://gepia2.cancer-pku.cn/囚to analyse the gene expression data and overall survival . Differentially expressed genes data analyses, we used the Omcomine databases (http://www.oncomine.org/) and the TIMER databases (http://www.timer.cistrome.org/), were performed using R software.

\section{Histological analysis and immunohistochemistry (IHC)}

For hematoxylin and eosin (H\&E) staining (G1005, Servicebio), glioma tissues were fixed with $10 \%$ neutral formalin for $24 \mathrm{~h}$, dehydrated, embedded in paraffin, and then cut into 5 - $\mu \mathrm{m}$-thick sections. After being dewaxed and treated with gradient alcohol, the sections were stained with hematoxylin for 5 min at room temperature and washed with running water. Subsequently, an alcohol solution with $1 \%$ hydrochloric acid and ammonia was employed. Eosin (1\%) was utilized for further staining for $3 \mathrm{~min}$ at room temperature, gradient ethanol was used for section dehydration and neutral balsam for section mounting, followed by microscopic visualization (Nikon, Japan).

IHC Sections of formalin-fixed paraformaldehyde-embedded cancerous glioma tissues were dewaxed and rehydrated. The endogenous peroxides were quenched by $0.3 \%$ hydrogen peroxide. These sections were initially incubated with primary antibodies and then with horseradish peroxidase (HRP)-conjugated secondary antibodies. Finally, the signal in various sections was analyzed using diaminobenzidine. After the sections were sealed with neutral glue, images were obtained using a microscope (Nikon, Japan).

\section{RNA sequencing analysis}

T98G cells were transfected with sh-NC $(n=3)$ or sh-LINC01578\#2 $(n=3)$ for 48 h. Then, total RNA was extracted using TRIzol reagent (Ambion, life technologies, Carlsbad, CA, USA) according to the manufacturer's instructions. Perform transcriptome sequencing, extract the total RNA of the sample and digest the DNA with DNase, use magnetic beads with Oligo (dT) to enrich the eukaryotic mRNA, add the interrupting reagent to interrupt the mRNA into short fragments to interrupt the mRNA is used as a template for PCR amplification, after the constructed library is qualified with Agilent 2100 Bioanalyzer(Agilent Technologies, Inc.), it is sequenced using sequencers such as Illumina HiSeqTM 2500 
or Illumina HiSeq X Ten(SF, USA). Significantly differentially expressed genes (DEGs) between sh-NC and sh-LINC01578\#2 cells were identified based on fold change $\geq 1.5$ and P-value $\leq 0.05$ using the DEGseq functions. The transcriptome sequencing and analysis were conducted by OE biotech Co., Ltd. (Shanghai, China).

\section{Statistical analysis}

Data are presented as the mean \pm SD of three independent experiments. All statistical were calculated in GraphPad Prism 6 by Student's $t$ test to compare the data. One-way analysis of variance followed was used to compare the difference among different groups. Differences were considered statistically significant at $* p<0.05,{ }^{*} p<0.01$ and ${ }^{* \star *} p<0.001$.

\section{Results}

\section{LINC01578 was significantly upregulated in the gliomas}

To examine the function of IncRNA in glioma tumorigenesis, we obtained the genome wide expression from TCGA databases (http://tcga-data.nci.nih.gov/tcga/). We found LINC01578 exhibited significant upregulation in the glioma tissues compared to the normal tissues (Fig. 1A and Additional file: Table S1). In GEPIA2 database (http://gepia2.cancer-pku.cn/), the LINC01578 was highly expressed in GBM (glioblastoma multiforme) and LGG (low grade glioma) (Additional file 1: Fig. S1A). And high LINC01578 expression correlated with poorer overall survival in glioma patients(Additional file 1: Fig. S1B). To confirm the databases results, we performed RT-qPCR to quantify the expression levels of LINC01578 in brain tissues and cell lines. Compared with normal tissues, LINC01578 expression was upregulated in glioma tissues. LINC01578 was overexpression in glioma cell lines (Fig. 1B-C). Fluorescence in situ hybridization (FISH) indicated that LINC01578 mainly located in the cytoplasm (Fig. 1D).

\section{LINC01578 knockdown decreases proliferation and metastasis}

The RT-qPCR shown that the shLINC01578\#2 exhibited the higher knockdown effect in T98G and A172 cells (Additional files: Fig S2). EdU and colony formation assays indicated that LINC01578 knockdown significantly inhibited the proliferation of glioma cells (Fig. 2A-B). Wound-healing assay demonstrated migration of glioma cells was significantly suppressed when LINC01578 was downregulated. Transwell analysis confirmed that LINC01578 knockdown inhibited the migratory and invasive potential of glioma cells (Fig. 2C-E). These results suggesting that LINC01578 may regulate proliferation and metastasis. Western blot analysis was used to detect the proliferation and metastasis related protein. Proliferating cell nuclear antigen (PCNA) plays an essential role in the proliferation process [20]. Cyclin D1 and E1 are important regulator of cell cycle [21-22]. In LINC01578-silenced cells, expression of PCNA, Cyclin D1 and E1 were decreased. Then we determined the expression of MMP2, MMP9, E-cadherin and N-cadherin which is required for tumor metastasis [23-26]. We found that MMP2, MMP9, N-cadherin was significantly 
downregulated, whereas the E-cadherin were upregulated with reduced LINC01578 (Fig. 2F). Collectively, our data suggested that LINC01578 function on proliferation囚migration and invasion of glioma cells.

\section{LINC01578 targets miR-6893-3p in glioma}

As LINC01578 is mainly located in the cytoplasm, LINC01578 might exert its effects via targeting miRNAs. RIP assays showed that LINC01578 binds to Ago2 (Fig.3A), the core component of microRNA (miRNA)-induced silencing complex [27]. These results indicated that LINC01578 may functions as a ceRNA and sponges miRNAs. To confirm this hypothesis, 4 bioinformatics databases (miRanda, TargetScan, RNAhybrid and starBase) were used to predict the potential interaction between LINC01578 and miRNAs. Through the database, we identified 5 miRNA contains binding sites (Fig. 3B and Additional file: Table S2). These results indicated that LINC01578 may act as the miR-6893-3p sponge.

To confirm the potential binding, a luciferase reporter assay was performed. In the 293T tool cell LINC01578-wt group, miR-6893-3p mimics significantly reduced the luciferase activity, and miR-6893-3p inhibitor significantly increased the luciferase activity. However, the luciferase activity was unchanged in LINC01578-mut group which putative miR-6893-3p binding site in the LINC01578 sequence was mutated, the same trend of luciferase reporter gene determination in A172 cell line (Fig. 3C). The FISH assays shown LINC01578 was colocalized with miR-6893-3p in the cytoplasm (Fig. 3D). These results suggest that LINC01578 functions as a miR-6893-3p sponge in glioma cells. In order to detected the expression level of miR-6893-3p, we performed RT-qPCR to quantify the expression levels of miR-6893-3p in brain tissues and cell lines. Compared with normal tissues, miR-6893-3p expression was downregulated in glioma tissues. miR-6893-3p was low expressed in glioma cell lines (Fig. 3E-F). We investigated the expression levels of miR-6893-3p in glioma cells transfected with miR-6893-3p mimics or inhibitor, the transfection efficiency of glioma cell lines was successful (Additional files: Figure S3).

\section{LINC01578 targets miR-6893-3p to exert its function in glioma}

To investigate the interactions among LINC01578 and miR-6893-3p on the progression of glioma, rescue experiments were performed. We knockdown the expression of miR-6893-3p in LINC01578-silenced glioma cells and detected the proliferation $\bigotimes$ migration and invasion in glioma cells. The EdU and colony formation assays indicated that miR-6893-3p inhibitor suppressed the decreased cell proliferation ability in LINC01578 knockdown glioma cells (Figure 4A and B). Furthermore, reduction effect of LINC01578 on cell migration and invasion was partially eliminated by miR-6893-3p knockdown (Fig. 4C). Similarly, the effects of down-regulation of PCNA, Cyclin D1 and E1, MMP2, MMP9, N-cadherin proteins and upregulation of E-cadherin protein in LINC01578 knockdown cells were reversed by miR-6893-3p inhibitor (Fig. 4D). These results suggest that LINC01578 affects glioma cells proliferation and metastasis by sponging miR-6893-3p.

\section{TRIM14 is a direct target of miR-6893-3p in glioma}


To determine the targets of miR-6893-3p regulated by LINC01578, RNA sequencing was used to analysis the differentially expressed genes in LINC01578 knockdown cells. 904 genes were downregulated and 144 genes were upregulated in shLINC01578 group compared to NC group (Fig. 5A and Additional file: Table S3). Then take the intersection of the 5 miRNA target genes (miRanda, TargetScan, RNAhybrid bioinformatics databases were used to predict the target genes) and the list of differentially expressed genes in the LINC01578 knockdown cells analyzed by RNA sequencing in Figure 5A (Fig. 5B and Additional file: Table S4). We constructed a ceRNA network with the downregulated genes and target genes of miR-6893-3p (Fig. 5C and Additional file: Table S5). We selected TRIM14 that have obvious differences and may have potential functions in the progression of glioma for research. Many studies have shown that TRIM family proteins play important roles in various physiological processes, including cell proliferation, migration, invasion, apoptosis and differentiation, and cell cycle. Therefore, TRIM14 was selected as a putative target of miR-6893-3p regulated by LINC01578 for further research.

The dual-luciferase reporter assay was used to validated their relationship. In the 293T tool cell TRIM14wt group, miR-6893-3p mimics significantly reduced the luciferase activity, and miR-6893-3p inhibitor significantly increased the luciferase activity. However, the luciferase activity was unchanged in TRIM14mut group which putative miR-6893-3p binding site in the TRIM14 sequence was mutated. The same trend of luciferase reporter gene determination in A172 cell line (Fig. 5D). We investigated the expression levels of LINC01578 in glioma cells transfected with overexpression LINC01578, The transfection efficiency of glioma cell lines was successful (Additional files: Fig S4a). In glioma cells, RT-qPCR and western blot results showed TRIM14 expression was decreased when LINC01578 was inhibited. TRIM14 expression was increased when LINC01578 was overexpressed (Additional files: Figure S4B-C). RT-qPCR and western blot results showed TRIM14 expression was decreased when miR-6893-3p was overexpressed. TRIM14 expression was increased when miR-6893-3p was inhibited (Additional files: Figure S4D-E). The GEPIA2 database shown TRIM14 was highly expressed in glioma tissues (Fig. 5E). The TCGA database shown that the higher the WHO grade of glioma patients, the higher the expression of TRIM14 (Fig. 5F and Additional file: Table S6). In addition, receiver operating characteristic (ROC) curve analysis was determined to evaluate the diagnostic value of LINC RNA 01578 for glioma (Fig. 5G and Additional file: Table S7).

TRIM14 mRNA expression levels were analyzed in Oncomine to examine TRIM14 expression over a cancer-wide range (Additional files: Fig. S5A), the results revealed that compared with that in the normal groups, TRIM14 expression was higher in brain and CNS cancer groups. To further evaluate TRIM14 expression in pan-cancer, we examined RNA sequencing data in TCGA using TIMER. The differential TRIM14 expression patterns in tumor and adjacent normal tissues are shown in Additional files: Fig. S5B and Additional file: Table S8. Potential interactions between TRIM14 and other genes were analyzed using GeneMANIA (http://genemania.org). (Additional files: Fig. S5C and Additional file: Table S9). The GEPIA2 database shown higher TRIM14 expression levels were significantly correlated with shorter overall survival (Additional files: Fig. S5D). Subsequently, Kaplan-Meier survival curves all WHO grade glioma patients and overall survival in all patients with glioma from the TCGA database showed that the overall survival of glioma patients with high levels of TRIM14 were shorter than patients with low levels 
of LINC01578 expression(Additional file 1: Fig. S5E and Additional file: Table S10).Our RT-qPCR results showed TRIM14 was remarkably increased in glioma tissues and cells (Fig. $5 \mathrm{H}-\mathrm{I})$. Our western blot results showed TRIM14 was remarkably increased in glioma cells (Fig. 5J).

\section{TRIM14 contributes to the function of miR-6893-3p}

The RT-qPCR and western blot shown that the si-TRIM14\#1 exhibited the higher knockdown effect in T98G and A172 cells (Additional files: Figure S6A-B). To further investigate miR-6893-3p that affects glioma cell by regulating TRIM14, we proved that TRIM14 knockdown inhibited the proliferation, colony formation, migration. (Fig. 6A-C). Then, we explored whether miR-6893-3p downregulation counteracted the supression of cell phenotypes caused by TRIM14 knockdown in glioma cells. In the T98G and A172 cells, inhibition of cell phenotypes by TRIM14 knockdown was counteracted by downregulation of miR6893-3p. Taken together, these results that miR-6893-3p affects glioma cell proliferation, migration, and invasion by regulating TRIM14 expression.

\section{LINC01578 promote the Akt/mTOR/P70S6K pathway}

To determine the potential mechanism of how LINC01578 regulated the migration and invasion of glioma cells, we performed western blot analysis to investigate the effects of IncRNA LINC01578 knockdown on the Akt/mTOR signaling pathway, which is often plays an important role in the progression of human cancer tumor cells. To investigate whether LINC01578 regulate the Akt/mTOR/P70S6K pathway by miR6893-3p/TRIM14 axis, we determined the expression of phosphorylated Akt/mTOR/P70S6K. LINC01578 overexpression promoted the expression of the phosphorylated forms of Akt, mTOR, P70S6K, but inhibited PTEN expression. While the phosphorylation of Akt, mTOR, P70S6K was suppressed in miR6893-3p-overexpressed cells (Fig. 7A-B). Further study shown sh-LINC01578-mediated inactivation of the Akt/mTOR/P70S6K signaling pathway was reversed upon co-transfection with miR-6893-3p inhibitor (Fig. 7C). The phosphorylation of Akt, mTOR, P70S6K was suppressed in TRIM14-silenced cells (Fig. 7D).

\section{LINC01578 facilitates glioma growth in vivo}

A xenograft mouse model was used to examine the function of LINC01578 on glioma growth in vivo. Compared with NC group, the tumor weight and volume in LINC01578 knockdown group was significantly inhibited (Fig. 8A-C). We then detected the TRIM14 expression in the tumors of xenograft mouse model by RT-qPCR and western blot. The expression level of TRIM14 in LINC01578 knockdown group was decreased (Fig. 8D-E).

\section{$\mathrm{m}^{6} \mathrm{~A}$ modification is involved in the upregulation of LINC01578 in glioma}

The epigenetic mechanisms of $\mathrm{m}^{6} \mathrm{~A}$ modification responsible for the upregulation of LINC01578 in glioma cells were investigated. $\mathrm{m}^{6} \mathrm{~A}$ RIP $q \mathrm{PCR}$ showed $\mathrm{m}^{6} \mathrm{~A}$ modification of LINC01578 was highly existed in T98G and A172 cells (Fig. 9A). METTL3 is defined as the main methyltransferase of the $\mathrm{m}^{6} \mathrm{~A}$ modification and involved in glioma development [28-29]. The RT-qPCR and western blot shown that the 
si-METTL3\#1 exhibited the higher knockdown effect in T98G and A172 cells (Additional files: Fig. S6). We found that METTL3 knockdown inhibited LINC01578 expression in glioma cells. Meanwhile, $\mathrm{m}^{6} \mathrm{~A}$ modification of LINC01578 was decreased in METTL3-silenced glioma cells (Fig.9A-B). To further clarify mettl3-meidated $\mathrm{m}^{6} \mathrm{~A}$ modification on LINC01578 expression, we predicted A RRACU $\mathrm{m}^{6} \mathrm{~A}$ sequence motifs of LINC01578 from 3 bioinformatics database (RFAthM6A, SRAMP, BERMP) (Fig. 9C and Additional file: Table S11.). Next, we constructed a mutant form of LINC01578, in which the adenine residues in the $\mathrm{m}^{6} \mathrm{~A}$ motifs were replaced with guanine (A-G transition mutation) (Fig. 9D). Luciferase reporter assay revealed that transcriptional level of wild-type LINC01578, but not the mutation, significantly decreased in the METTL3 knockdown group (Fig. 9E). By treating glioma cells with actinomycin-D to inhibit transcription, the half-life of LINC01578 was decreased in METTL3-silenced cells (Fig. 9F). These data suggested that METTL3-mediated $\mathrm{m}^{6} \mathrm{~A}$ is associated with the upregulation of LINC01578, probably by regulating the stability of its transcript. According to the RMBase v2.0 database [30], YTHDF1 is an RNA-binding protein (RBP) of LINC01578. YTHDF protein can directly bind to and recognize $m^{6}$ A-modified IncRNAs. RIP qPCR assay shown that YTHDF1 directly bonded to LINC01578 to form an m6A modification complex (Fig. 9G). RT-qPCR showed that the inhibitory efficiency of YTHDF1 in glioma cell lines proved that the inhibition was successful (Fig. 9H). Moreover, RT-qPCR indicated that expression of LINC01578 was decreased in YTHDF1-silenced cells (Fig. 9I). In conclusion, these results indicating that METTL3 mediate $\mathrm{m}^{6} \mathrm{~A}$ modification of LINC01578, YTHDF1 selectively binds to $\mathrm{m}^{6} \mathrm{~A}$ modifed LINC01578 and modulates LINC01578 expression.

\section{Discussion}

Accumulating evidence has demonstrated that many dysregulated IncRNAs in glioma are closely related to tumorigenesis, metastasis, and prognosis or therapies[31]. In this study, a TCGA and Gepia databases was used to screen the differentially expressed IncRNAs in glioma and LINC01578 was upregulated in glioma. Highly expressed LINC01578 also associated with a poor prognosis of glioma patients. However, the function and molecular mechanism of LINC01578 in glioma remain need further study. Functional experiments in vivo and in vitro demonstrated that LINC01578 regulated glioma tumorgenesis, such as proliferation, migration, invasion, and tumor growth.

Since the functional mechanism of IncRNAs depends on their subcellular localization, IncRNAs can also be grouped into nuclear or cytoplasmic categories [32]. Most of LINC01578 is in cytoplasm, suggesting that LINC01578 may serve as a miRNA sponge. We defined the miR-6893-3p as the targets of LINC01578 by using bioinformatics databases, RIP, dual luciferase reporter, and FISH assays. Further functional experiments showed that LINC01578 sponged miR-6893-3p to promote glioma progression. The miR6893-3p which has less research was found upregulated in the serum multiple myeloma (MM) [33].

A comprehensive analysis combined RNA sequencing results and bioinformatics databases was used to investigate the target gene of the miR-6893-3p regulated by LINC01578 in glioma. The ceRNA network indicate that TRIM14 was the downstream effector of the LINC01578/miR-6893-3p axis. TRIM family 
proteins play important roles in various physiological processes, including cell proliferation, migration, invasion, apoptosis and differentiation, and the cell cycle [34]. TRIM14 was upregulated in human glioblastoma and functions as a novel regulator of EMT by controlling the abundance of ZEB2 [35]. TRIM14 also play a specific biological role of in temozolomide resistance of glioma cells [36-37]. Functional experiments showed that TRIM14 contributes to the function of miR-6893-3p in glioma.

Gathering evidence has revealed that Akt signaling pathway plays an important role in glioma progression and aggressiveness [38]. Therefore, targeting Akt could provide an important approach for glioma prevention and therapy [39]. KEGG enrichment show that Akt signaling pathway was downregulated following LINC01578 knockdown in glioma. Our results revealed that LINC01578 acts as a tumour promote via the miR-6893-3p to active the Akt signaling pathway in glioma. TRIM14 regulates cell proliferation and invasion in osteosarcoma and melanoma via promotion of the PTEN/PI3K/Akt signaling pathway [40-41]. The Akt/mTOR/P70S6K pathway also affected by TRIM14 in glioma. Therefore, we hypothesized LINC01578/miR-6893-3p/TRIM14 axis promoted glioma cells proliferation, migration, and invasion via activating Akt/mTOR/P70S6K pathway.

$\mathrm{m}^{6} \mathrm{~A}$ has been demonstrated to affect the targeted IncRNA and participate in the progression of glioma. METTL3 enhances the stability of MALAT1 with the assistance of HuR via m6A modification in IDHwildtype glioma [29]. $\mathrm{m}^{6} \mathrm{~A}$-related IncRNAs are potential biomarkers for predicting the overall survival of glioma patients [42]. We revealed that $\mathrm{m}^{6} \mathrm{~A}$ methylation was enriched within LINC01578 in glioma cells using $\mathrm{m}^{6} \mathrm{~A}$ RIP experiment. Moreover, METTL3 regulated the $\mathrm{m}^{6} \mathrm{~A}$ modification in LINC01578, thus affecting its RNA stability. YTHDF3 facilitates translation of protein synthesis in synergy with YTHDF1 and affects decay of methylated mRNA mediated through YTHDF2 [43]. YTHDF3 selectively binds $\mathrm{m}^{6} \mathrm{~A}-$ methylated GAS5 to trigger its decay [13]. In our study, YTHDF1 selectively binds to $\mathrm{m}^{6} \mathrm{~A}$-modifed LINC01578 and modulates LINC01578 expression.

\section{Conclusion}

In summary, we have shown that LINC01578 is upregulated in glioma and High LINC01578 level was associated with the poor prognosis in glioma patients. LINC01578 play role on cell proliferation, migration, and invasion by modulating the miR-6893-3p/TRIM14 axis. LINC01578/miR-6893-3p/TRIM14 axis also regulates the Akt/mTOR/P70S6K pathway. Additionally, we provide evidence that METTL3 and YTHDF1 mediated $\mathrm{m}^{6} \mathrm{~A}$ modification was responsible for the upregulation of LINC01578 in glioma. Our study of LINC01578 may provide a prognostic predictor and therapy target for the treatment of glioma.

\section{Abbreviations}

\section{IncRNAs}

Long noncoding RNAs

RIP

RNA immunoprecipitation 
$\mathrm{m} 6 \mathrm{~A}$

N6-methyladenosine

WHO

World Health Organization

GBM

glioblastoma

TMZ

temozolomide

ncRNAs

non-coding RNAs

WTAP

Wilm's tumor protein

YTHDC1

YTH domain-containing 1

YTHDF1

YTH N6-methyladenosine RNA-binding protein 1

HEB

normal human glial cell line

DMEM

Dulbecco's modified eagle medium

FBS

fetal bovine serum

shRNA

short hairpin RNAs

FISH

Fluorescence in situ hybridization

WB

Western blotting

TCGA

the Cancer Genome Atlas database

IHC

immunohistochemistry

HRP

horseradish peroxidase

DEGs

differentially expressed genes

LGG

low grade glioma

\section{Declarations}




\section{Ethics approval and consent to participate}

This research protocol was approved by the Ethics Committee of the first affiliated Hospital of Wannan Medical College (No. 2020-08-05).

\section{Consent for publication}

Not applicable.

\section{Availability of data and materials}

The data used to support the findings of this study are available from the corresponding author upon request.

\section{Competing interests}

The authors declare that they have no competing interests.

\section{Funding}

This work was supported by grants from the National Natural Science Foundation of China (grant nos. 81802503 to H.Y. and 82072370 to K.L.), Natural Science Foundation of Anhui Province邓grant nos. 2008085QH367 to X.L.Z.), Funding of "Peak" Training Program for Scientific Research of Yijishan Hospital, Wannan Medical College(grant nos.GF2019G10 to X.L.Z. and GF2019G15 to H.Y.).

\section{Authors' Contributions}

$\mathrm{KL}$ designed the experiments. XWW, $\mathrm{HY}$ and $\mathrm{X} L Z$ performed the experiments and analyzed the data. $\mathrm{XWW}$ and $\mathrm{HY}$ wrote the manuscript. MZ and MZh contributed reagents/materials and animal housekeeping. All authors approved the final version of the manuscript.

\section{Acknowledgments}

We thank LetPub (www.letpub.com) for its linguistic assistance during the preparation of this manuscript.

\section{References}

1. Niu W, Xiao Q, Wang X, et al. A biomimetic drug delivery system by integrating grapefruit extracellular vesicles and doxorubicin-loaded heparin-based nanoparticles for glioma therapy. Nano Lett. 2021;21(3):1484-92.

2. Jin L, Shi F, Chun Q, et al. Artificial intelligence neuropathologist for glioma classification using deep learning on hematoxylin and eosin stained slide images and molecular markers. Neuro Oncol. 2021;23(1):44-52. 
3. Hasan MN, Luo L, Ding D, et al. Blocking NHE1 stimulates glioma tumor immunity by restoring OXPHOS function of myeloid cells. Theranostics. 2021;11(3):1295-309.

4. Li J, Liao T, Liu H, et al. Hypoxic glioma stem cell-derived exosomes containing linc01060 promote progression of glioma by regulating the MZF1/c-Myc/HIF1a axis. Cancer Res. 2021;81(1):114-28.

5. Sun J, He D, Fu Y, et al. A novel IncRNA ARST represses glioma progression by inhibiting ALDOAmediated actin cytoskeleton integrity. J Exp Clin Cancer Res. 2021;40(1):187.

6. Tang G, Luo L, Zhang J, et al. IncRNA LINC01057 promotes mesenchymal differentiation by activating NF-KB signaling in glioblastoma. Cancer Lett. 2021;498:152-64.

7. Li H, Yan R, Chen W, et al. Long non coding RNA SLC26A4-AS1 exerts antiangiogenic effects in human glioma by upregulating NPTX1 via NFKB1 transcriptional factor. FEBS J. 2021;288(1):21228.

8. Sheng J, He X, Yu W, et al. p53-targeted IncRNA ST7-AS1 acts as a tumour suppressor by interacting with PTBP1 to suppress the Wnt/ $\beta$-catenin signalling pathway in glioma. Cancer Lett. 2021;503:5468.

9. Rom A, Melamed L, Gil N, et al. Regulation of CHD2 expression by the Chaserr long noncoding RNA gene is essential for viability. Nat Commun. 2019;10(1):5092.

10. Liu J, Zhan Y, Wang J, Wang J, Guo J, Kong D. Long noncoding RNA LINC01578 drives colon cancer metastasis through a positive feedback loop with the NF-KB/YY1 axis. Mol Oncol. 2020;14(12):3211-33.

11. Ma S, Chen C, Ji X, et al. The interplay between $\mathrm{m}^{6} \mathrm{~A}$ RNA methylation and noncoding RNA in cancer. J Hematol Oncol. 2019;12(1):121.

12. Chen $X, X u M, X u X$, et al. METTL14-mediated $N^{6}$-methyladenosine modification of SOX4 mRNA inhibits tumor metastasis in colorectal cancer. Mol Cancer. 2020;19(1):106.

13. Ni W, Yao S, Zhou Y, et al. Long noncoding RNA GAS5 inhibits progression of colorectal cancer by interacting with and triggering YAP phosphorylation and degradation and is negatively regulated by the $\mathrm{m}^{6} \mathrm{~A}$ reader YTHDF3. Mol Cancer. 2019;18(1):143.

14. Yoneda R, Ueda N, Uranishi K, Hirasaki M, Kurokawa R. Long noncoding RNA pncRNA-D reduces cyclin D1 gene expression and arrests cell cycle through RNA $\mathrm{m}^{6} \mathrm{~A}$ modification. J Biol Chem. 2020;295(17):5626-39.

15. Liu H, Xu Y, Yao B, Sui T, Lai L, Li Z. A novel $N^{6}$-methyladenosine $\left(\mathrm{m}^{6} \mathrm{~A}\right)$-dependent fate decision for the IncRNA THOR. Cell Death Dis. 2020;11(8):613.

16. Zhang S, Zhao BS, Zhou A, et al. $\mathrm{m}^{6} \mathrm{~A}$ Demethylase ALKBH5 Maintains Tumorigenicity of Glioblastoma Stem-like Cells by Sustaining FOXM1 Expression and Cell Proliferation Program. Cancer Cell. 2017;31(4):591-606.e6.

17. Cui $Q$, Shi $H, Y e P$, et al. $m^{6} A$ RNA Methylation Regulates the Self-Renewal and Tumorigenesis of Glioblastoma Stem Cells. Cell Rep. 2017;18(11):2622-34. 
18. Li F, Yi Y, Miao Y, et al. $N^{6}$-Methyladenosine Modulates Nonsense-Mediated mRNA Decay in Human Glioblastoma. Cancer Res. 2019;79(22):5785-98.

19. Zhu X, Chen T, Yang H, Lv K. Lactate induced up-regulation of KLHDC8A (Kelch domain-containing $8 \mathrm{~A})$ contributes to the proliferation, migration and apoptosis of human glioma cells. J Cell Mol Med. 2020;24(20):11691-702.

20. He XY, Pan XM, Jin MM, et al. Long non-coding RNA AK027294 promotes tumor growth by upregulating PCNA in gastric cancer. Eur Rev Med Pharmacol Sci. 2019;23(13):5762-9.

21. Shi Q, Li Y, Li S, et al. LncRNA DILA1 inhibits Cyclin D1 degradation and contributes to tamoxifen resistance in breast cancer. Nat Commun. 2020;11(1):5513.

22. Zhang C, Zhu Q, Gu J, Chen S, Li Q, Ying L. Down-regulation of CCNE1 expression suppresses cell proliferation and sensitizes gastric carcinoma cells to Cisplatin. Biosci Rep. 2019;39(6):BSR20190381.

23. Wu DM, Deng SH, Liu T, Han R, Zhang T, Xu Y. TGF- $\beta$-mediated exosomal Inc-MMP2-2 regulates migration and invasion of lung cancer cells to the vasculature by promoting MMP2 expression [published correction appears in Cancer Med. 2020 Jul;9(13):4876]. Cancer Med. 2018;7(10):51185129.

24. Cheng $X$, Yang $Y$, Fan Z, et al. MKL1 potentiates lung cancer cell migration and invasion by epigenetically activating MMP9 transcription. Oncogene. 2015;34(44):5570-81.

25. Wu Y, Yang $X$, Chen Z, et al. m6A-induced IncRNA RP11 triggers the dissemination of colorectal cancer cells via upregulation of Zeb1. Mol Cancer. 2019;18(1):87.

26. Osuka S, Zhu D, Zhang Z, et al. N-cadherin upregulation mediates adaptive radioresistance in glioblastoma. J Clin Invest. 2021;131(6):e136098.

27. Chen $Y$, Yang F, Fang E, et al. Circular RNA circAGO2 drives cancer progression through facilitating HuR-repressed functions of AGO2-miRNA complexes. Cell Death Differ. 2019;26(7):1346-64.

28. Visvanathan A, Patil V, Arora A, et al. Essential role of METTL3-mediated m6A modification in glioma stem-like cells maintenance and radioresistance. Oncogene. 2018;37(4):522-33.

29. Chang YZ, Chai RC, Pang B, et al. METTL3 enhances the stability of MALAT1 with the assistance of HuR via m6A modification and activates NF-KB to promote the malignant progression of IDHwildtype glioma. Cancer Lett. 2021;511:36-46.

30. Xuan JJ, Sun WJ, Lin PH, et al. RMBase v2.0: deciphering the map of RNA modifications from epitranscriptome sequencing data. Nucleic Acids Res. 2018;46(D1):D327-34.

31. Peng Z, Liu C, Wu M. New insights into long noncoding RNAs and their roles in glioma. Mol Cancer. 2018;17(1):61.

32. Chen LL. Linking long noncoding RNA localization and function. Trends Biochem Sci. 2016;41(9):761-72.

33. Li J, Zhang M, Wang $\mathrm{C}$. Circulating miRNAs as diagnostic biomarkers for multiple myeloma and monoclonal gammopathy of undetermined significance. J Clin Lab Anal. 2020;34(6):e23233. 
34. Hatakeyama S. Trim family proteins: roles in autophagy, immunity, and carcinogenesis. Trends Biochem Sci. 2017;42(4):297-311.

35. Feng S, Cai X, Li Y, Jian X, Zhang L, Li B. Tripartite motif-containing 14 (TRIM14) promotes epithelialmesenchymal transition via ZEB2 in glioblastoma cells. J Exp Clin Cancer Res. 2019;38(1):57.

36. Deng Y, Zhu H, Xiao L, Liu C, Meng X. Circ_0005198 enhances temozolomide resistance of glioma cells through miR-198/TRIM14 axis. Aging. 2020;13(2):2198-211.

37. Tan Z, Song L, Wu W, et al. TRIM14 promotes chemoresistance in gliomas by activating Wnt/ $\beta$ catenin signaling via stabilizing Dvl2 [published correction appears in Oncogene. Oncogene. 2018;37(40):5403-15.

38. Chautard E, Ouédraogo ZG, Biau J, Verrelle P. Role of Akt in human malignant glioma: from oncogenesis to tumor aggressiveness. J Neurooncol. 2014;117(2):205-15.

39. Le Rhun E, Preusser M, Roth P, et al. Molecular targeted therapy of glioblastoma. Cancer Treat Rev. 2019;80:101896.

40. Chen J, Huang L, Quan J, Xiang D. TRIM14 regulates melanoma malignancy via PTEN/PI3K/AKT and STAT3 pathways. Aging. 2021;13(9):13225-38.

41. Xu G, Guo Y, Xu D, et al. TRIM14 regulates cell proliferation and invasion in osteosarcoma via promotion of the AKT signaling pathway. Sci Rep. 2017;7:42411.

42. Tu Z, Wu L, Wang P, et al. N6-Methylandenosine-Related IncRNAs Are Potential Biomarkers for Predicting the Overall Survival of Lower-Grade Glioma Patients. Front Cell Dev Biol. 2020;8:642.

43. Shi $H$, Wang $X$, Lu Z, et al. YTHDF3 facilitates translation and decay of N6-methyladenosine-modified RNA. Cell Res. 2017;27(3):315-28.

\section{Supplementary}

Supplementary Data s1 to s11 are not available with this version.

\section{Figures}




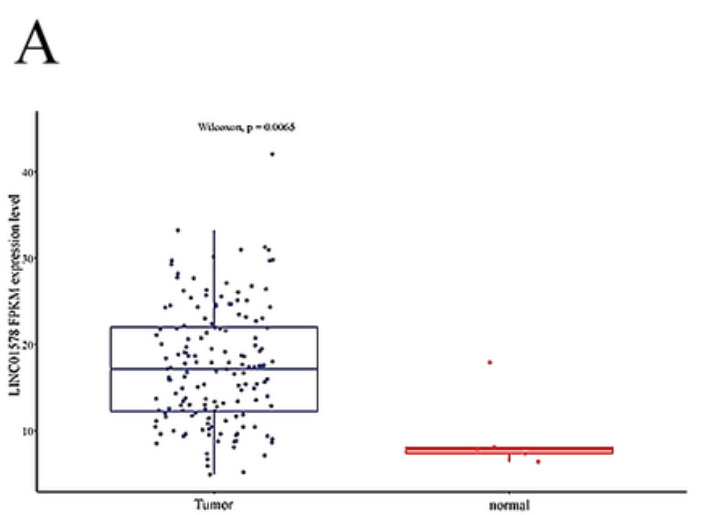

$\mathrm{B}$

C
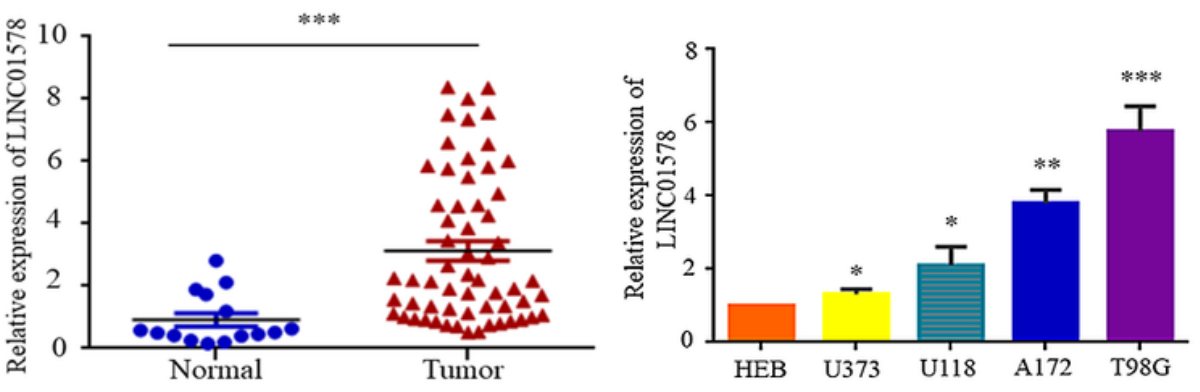

$\mathrm{D}$
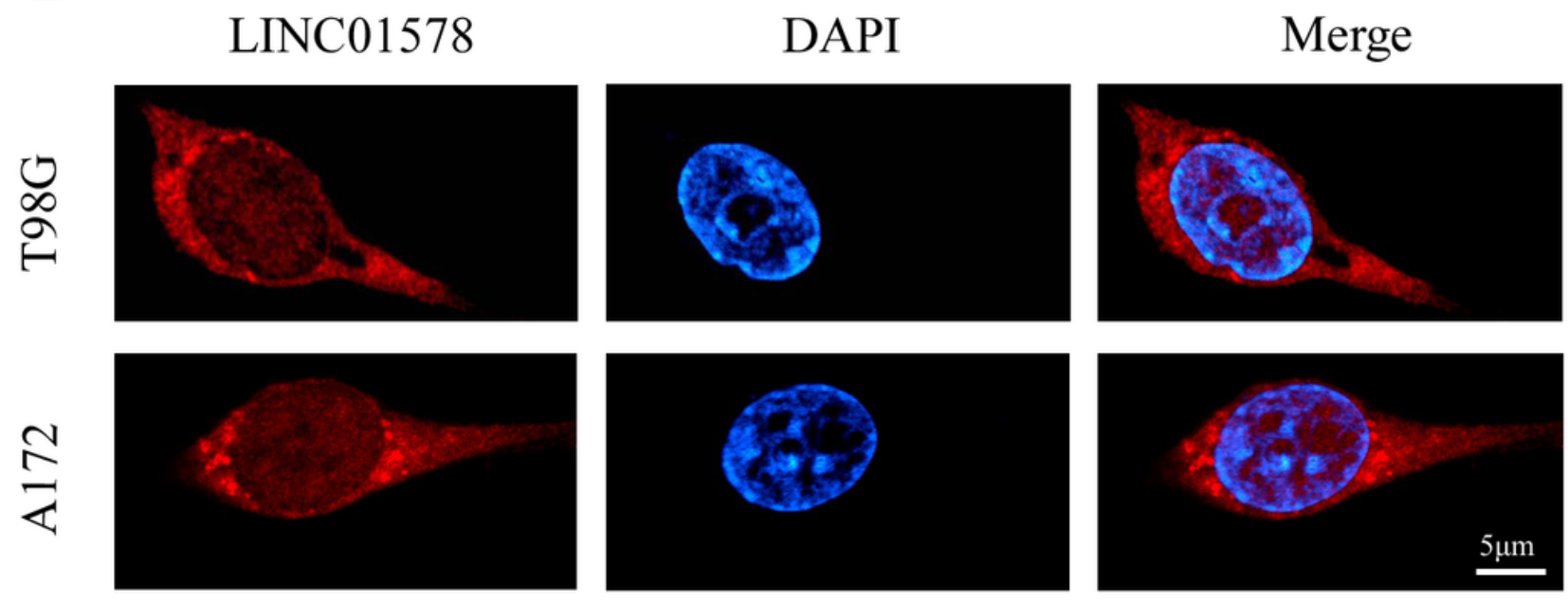

Figure 1

The expression and subcellular location of LINC01578 in glioma.

(A) The TCGA database showed that the expression level of LINC01578 in glioma was higher than that of a normal brain tissues. (B) Compared with normal brain tissues, LINC01578 was highly expressed in glioma tissues. LINC01578 expression in 66 glioma tissues and 14 normal brain tissues from Yijishan Hospital was detected by RT-qPCR. (C) RT-qPCR was used to detect the expression of LINC01578 in normal human glial cell line (HEB) and 4 human glioma cell lines (U373, U118, A172, T98G). (D) FISH assay was performed to determine the subcellular location of LINC01578 in T98G and A172 cells. (Normal and N: normal brain tissues; T: glioma; GBM: glioblastoma multiforme; LGG: low grade glioma),

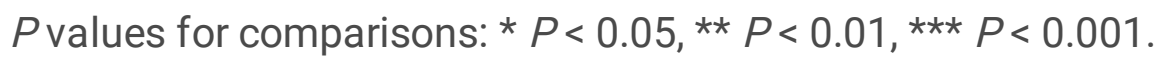




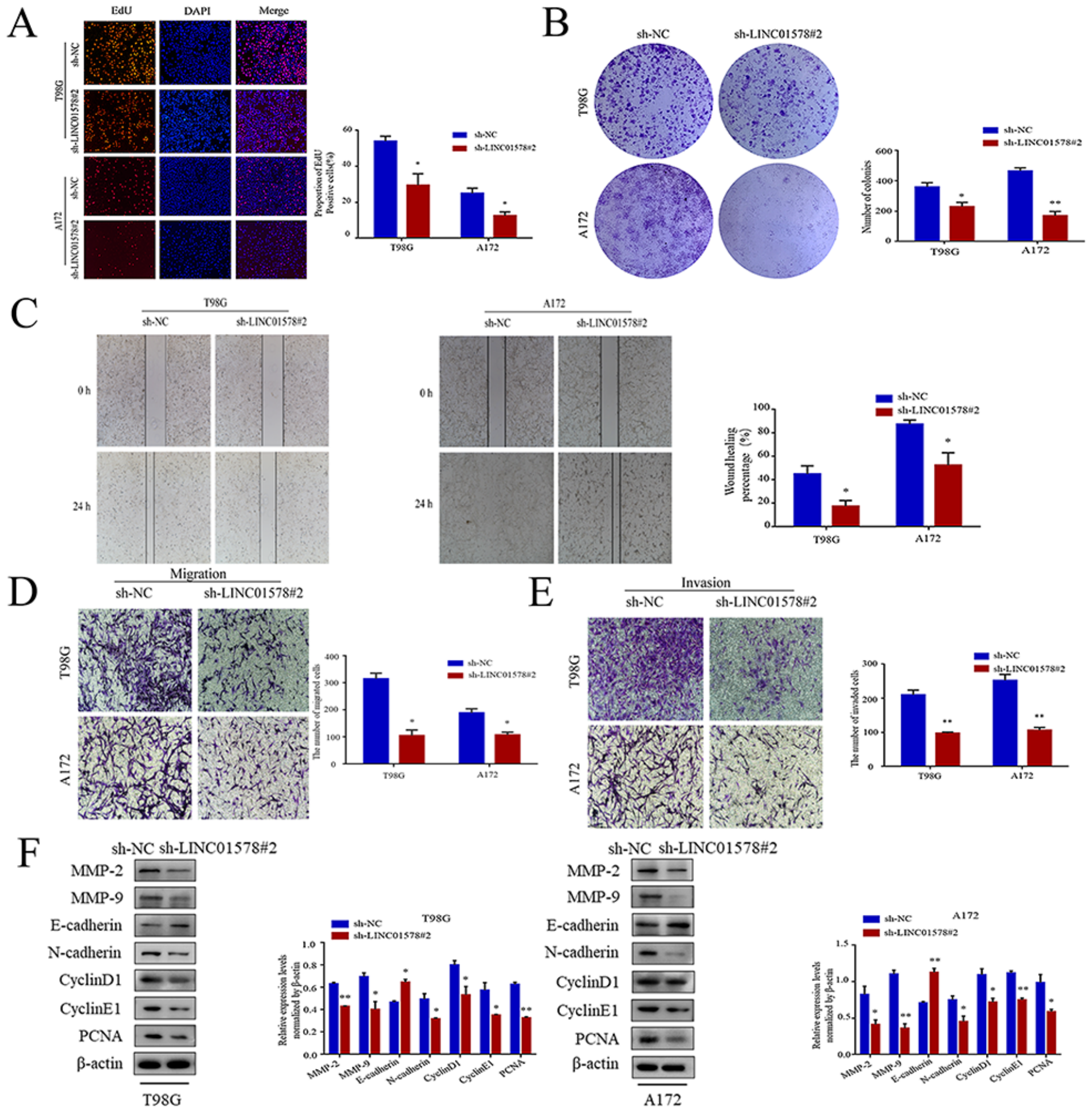

Figure 2

LINC01578 regulates glioma cell proliferation, migration, and invasion. (A) EdU assay was employed to determine the proliferative ability of T98G and A172 cells with LINC01578 knockdown. Percentage of EdU positive cells was quantified using the GraphPad. (B) Colony formation in T98G and A172 cells with LINC01578 knockdown was performed. Clone numbers was quantified using the GraphPad. (C) The migration of T98G and A172 cells with LINC01578 knockdown was determined by wound-healing assay. The wound recovery was analyzed using the GraphPad. (D-E) The migration and invasion of T98G and 
A172 cells with LINC01578 knockdown was determined by transwell assays. The migration and invasion cells were quantified using the GraphPad. (F) Western blot was used to detect the expression of indicated genes of T98G and A172 cells with LINC01578 knockdown. Data are mean \pm SD from three independent experiments. * $P<0.05, * \star P<0.01$, *** $P<0.001$.
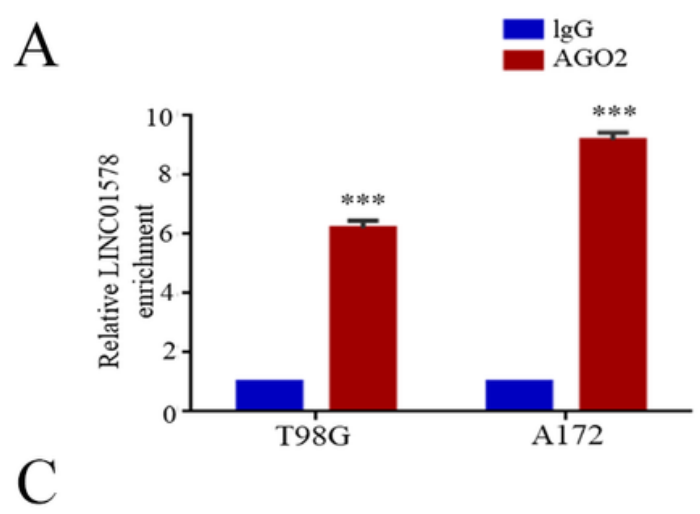

LINC01578

hsa-miR-6893-3p

LINC01578-MUT
5'----ATGGCCGGAG- AGGCAGCAGCA 3' \|\|$\||\||\|\mid\|$ 3'-----GACCGUCCACUUCCGUAGUCCC 5' 5'----ATCCGCCCAC- TCCGTCGTCCA 3'
B
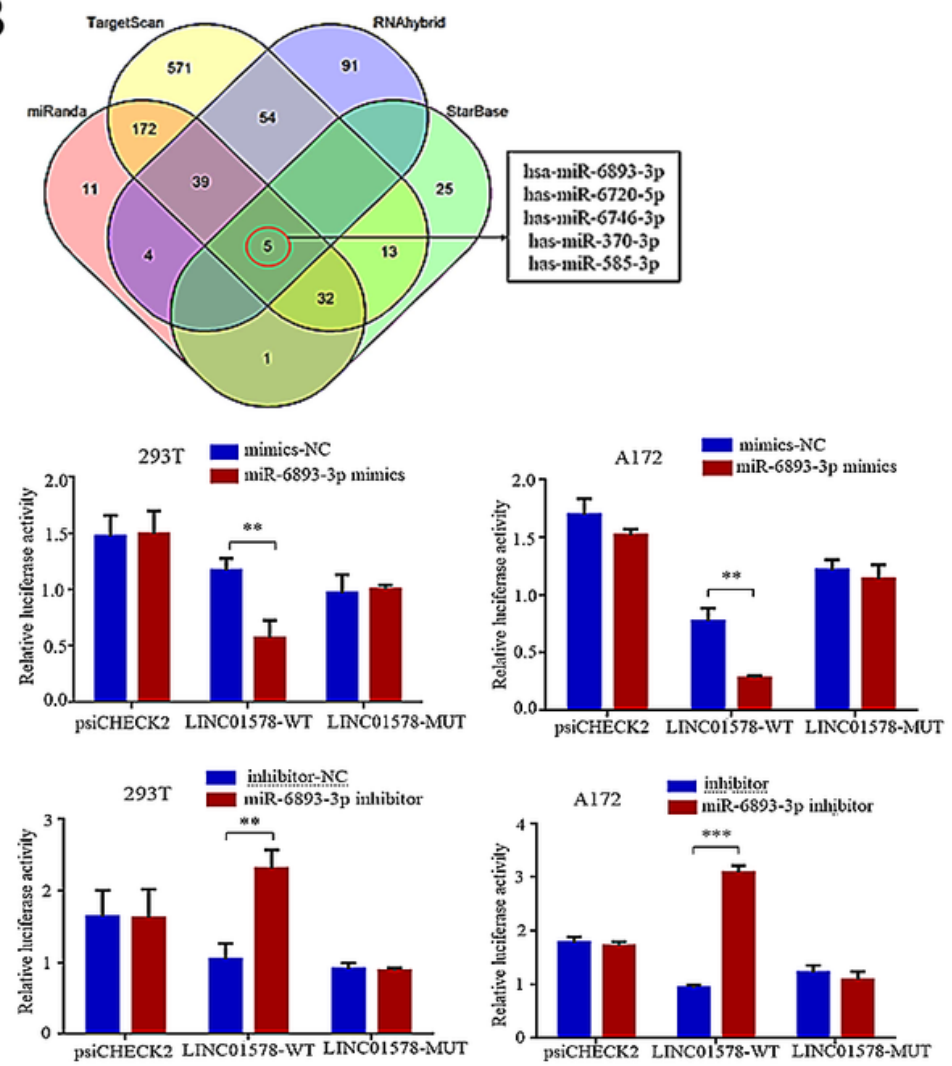

$\mathrm{E}$

$\mathrm{F}$
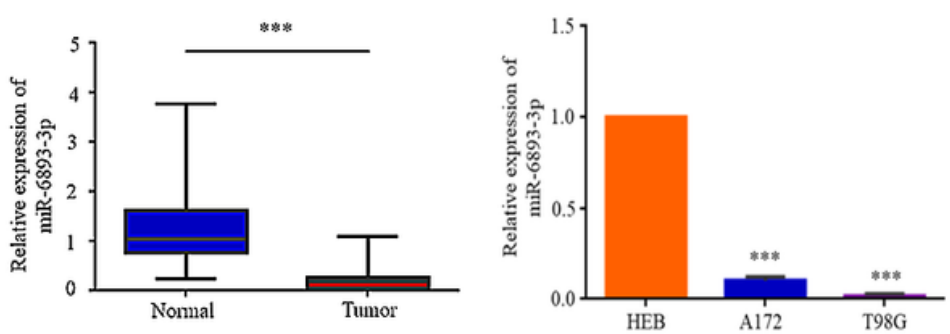

\section{Figure 3}

LINC01578 targets miR-6893-3p in glioma. (A) Anti-AG02 RIP and RT-qPCR were performed in T98G and A172 cells to detect the expression of LINC01578 associated with AGO2. (B) A total of 5 miRNAs were predicted to targets of LINC01578 according to miRanda, TargetScan, RNAhybrid and starBase databases. (C) The predicted binding sites between miR-6893-3p and LINC01578. Dual luciferase reporter assay was used to determine the luciferase activity in 293T and A172 cells co-transfected with wild-type (WT) or mutant (MUT) LINC01578 and miR-6893-3p mimics or miR-6893-3p inhibitor. (D) FISH assay was 
performed to detect the colocalization of LINC01578 and miR-6893-3p in cytoplasm in T98G and A172 cells. (E) RT-qPCR was used to detect the expression of miR-6893-3p in normal human brain tissues and human glioma tissues. (F) RT-qPCR was used to detect the expression of miR-6893-3p in normal human brain cell line (HEB) and 2 human glioma cell lines (T98G, A172). Compared with normal brain tissues, miR-6893-3p was lowly expressed in glioma tissues. Data are mean \pm SD from three independent experiments. $* \star P<0.01, * \star \star ~ P<0.001$.

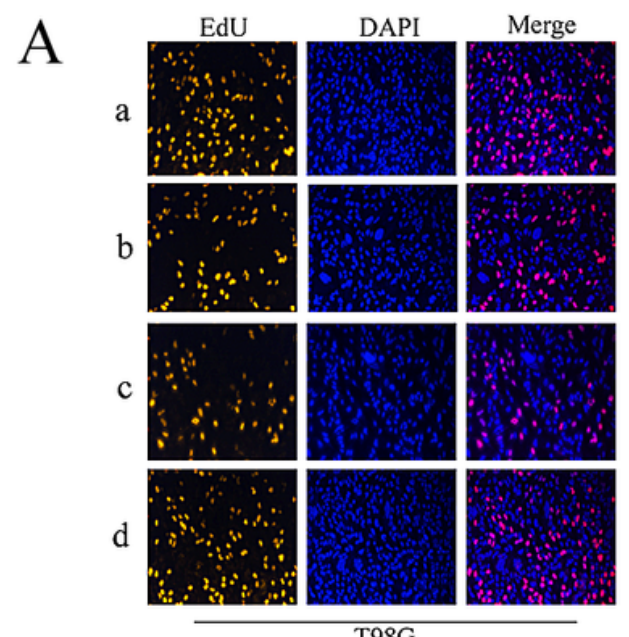

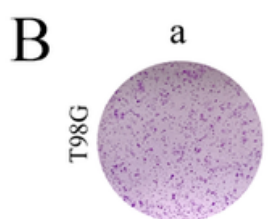

a

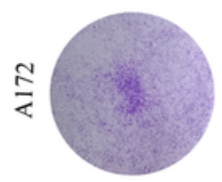

$\mathrm{b}$

$\mathrm{b}$
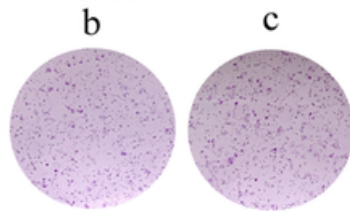

c

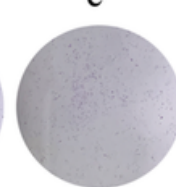

$\mathrm{C}$

a

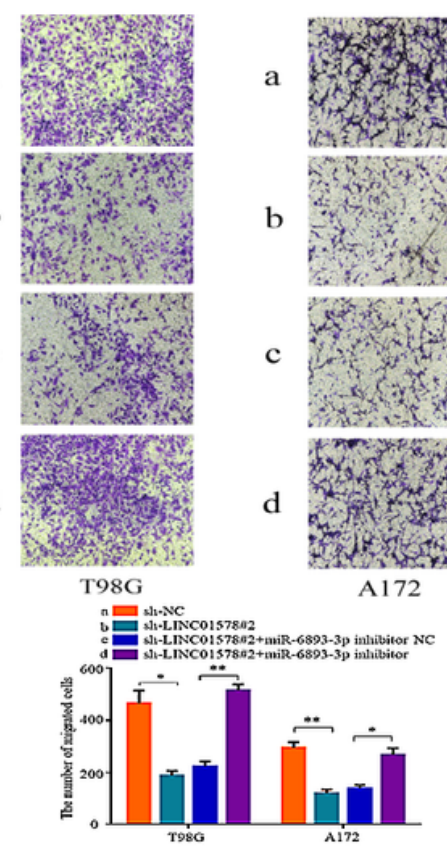

a

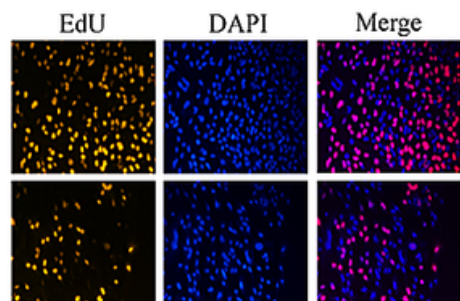

c

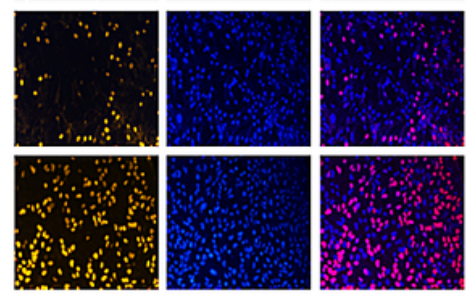

A172
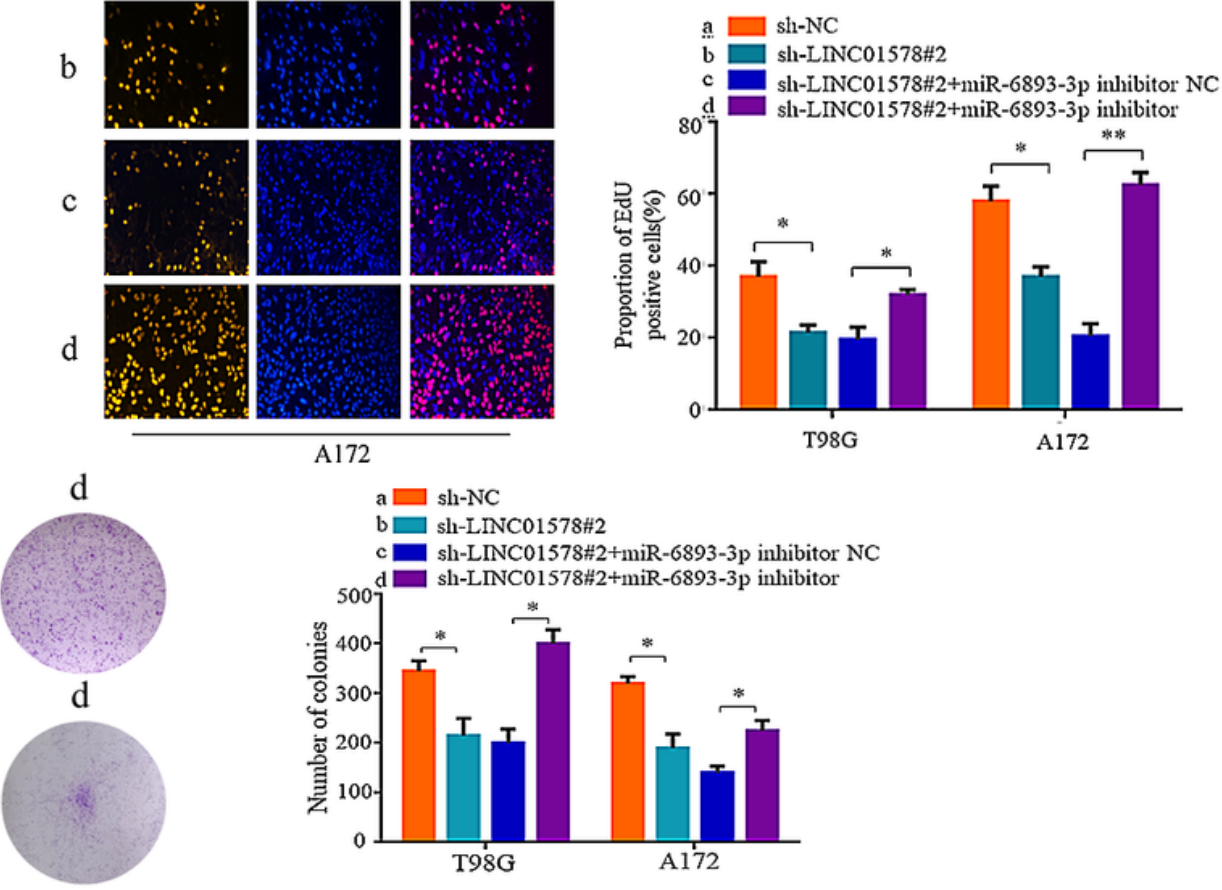

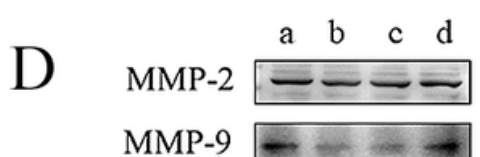

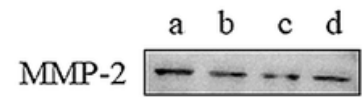

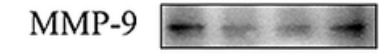

MMP-9

E-cadherin $-\longrightarrow$

$\mathrm{N}$-cadherin $-\cdots$

CyclinD1 $-\cdots$

CyclinE1 --

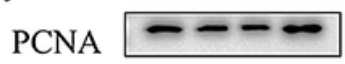

$\beta$-actin

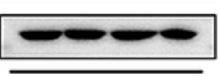

T98G

r9sa

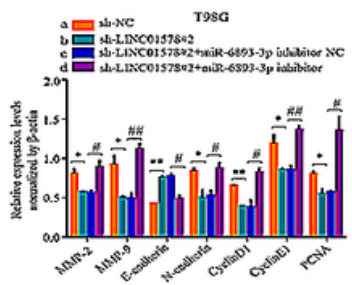

E-cadherin $\longrightarrow-\cdots$

$\mathrm{N}$-cadherin $-\cdots$

Cyclind 1 : $=$ E

CyclinE1 - - -

PCNA

$\beta$-actin $\longrightarrow-$

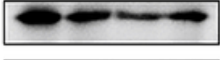

A172

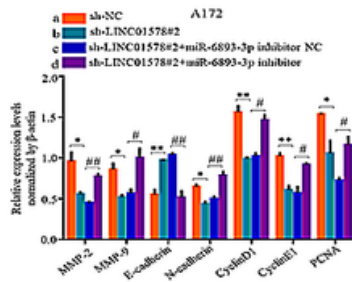

Figure 4 
LINC01578 targets miR-6893-3p to exert its function in glioma. (A) EdU assay was employed to determine the proliferative ability of T98G and A172 cells co-transfected with shLINC01578 and/or miR-6893-3p inhibitor. Percentage of EdU positive cells was quantified using the GraphPad. (B) Colony formation in T98G and A172 cells co-transfected with shLINC01578 and/or miR-6893-3p inhibitor was performed. Clone numbers was quantified using the GraphPad. (C) The migration of T98G and A172 cells cotransfected with shLINC01578 and/or miR-6893-3p inhibitor was determined by transwell assays. The migration cells were quantified using the GraphPad. (D) Western blot was used to detect the expression of indicated genes of T98G and A172 cells co-transfected with shLINC01578 and/or miR-6893-3p

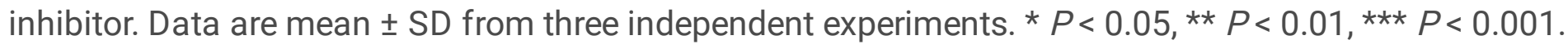


A

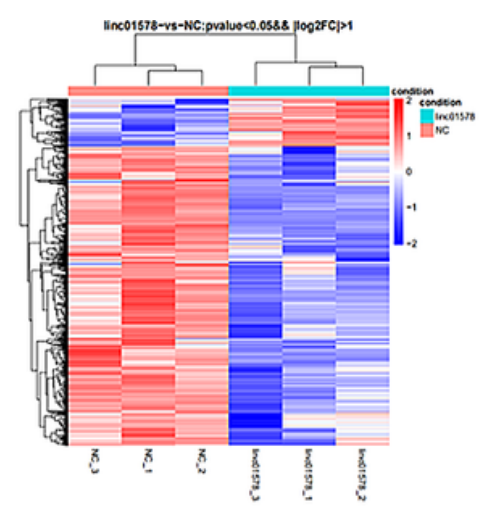

$\mathrm{D}$

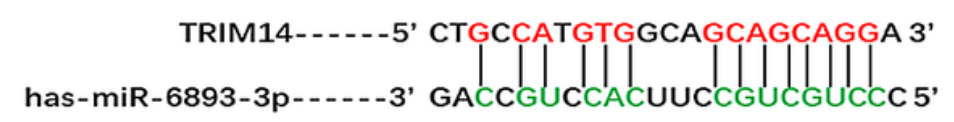

TRIM14-MUT--:---5' CTCCGTTCACGCACGTCGTCCA 3'

$\mathrm{E}$

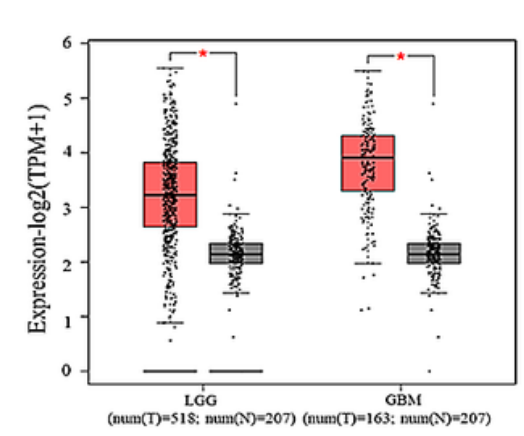

$\mathrm{H}$

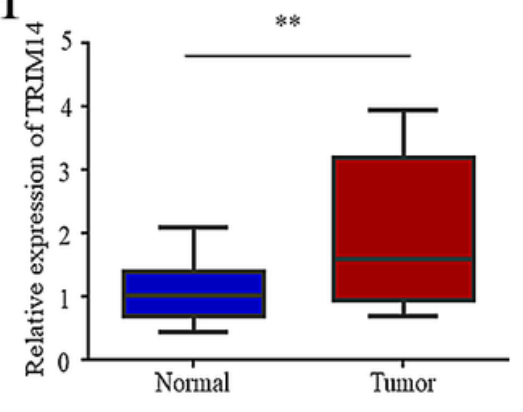

$\mathrm{B}$
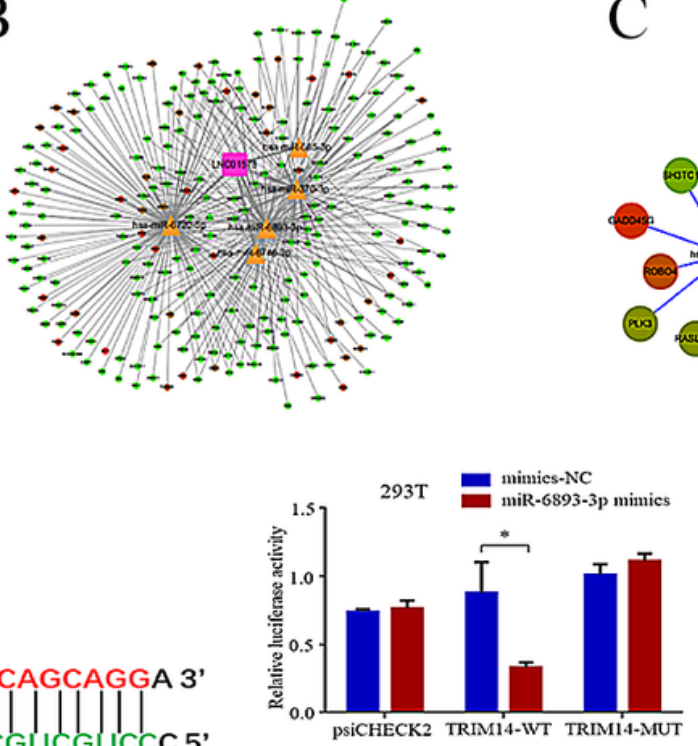

293T
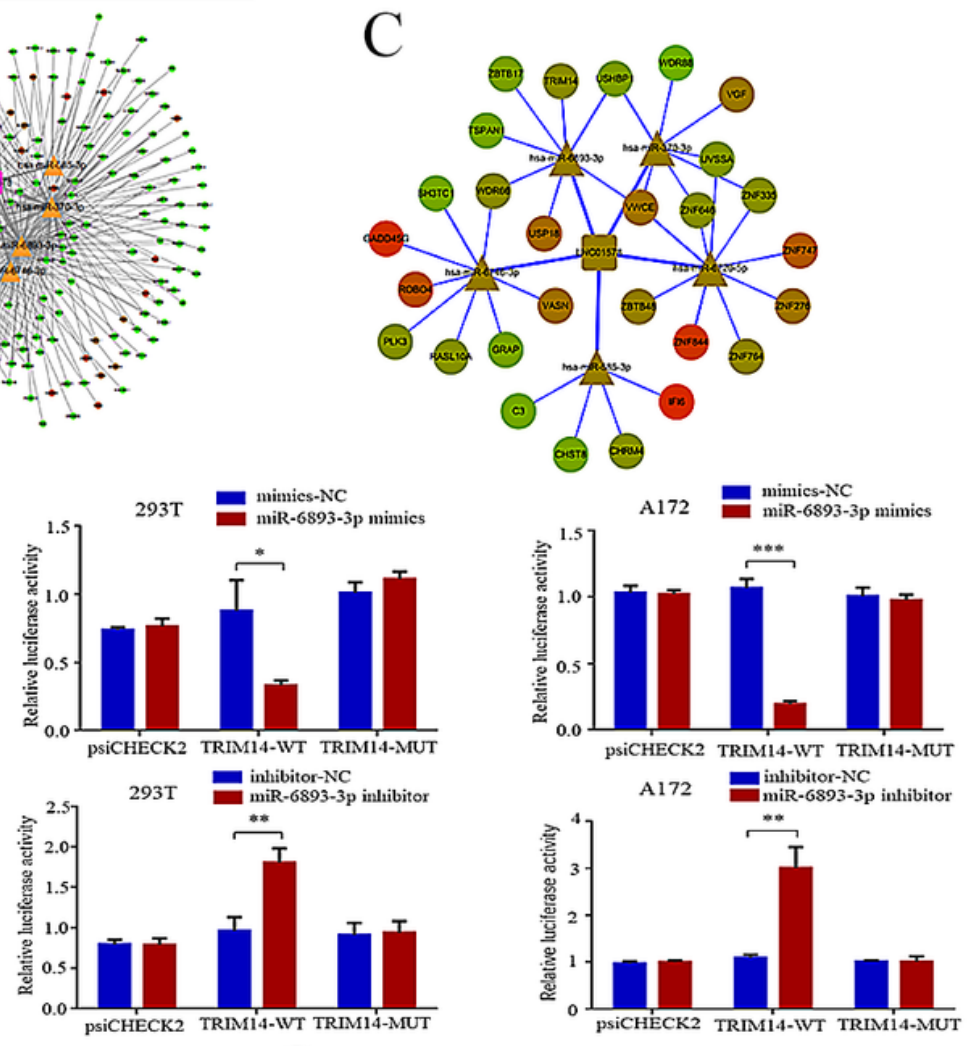

G
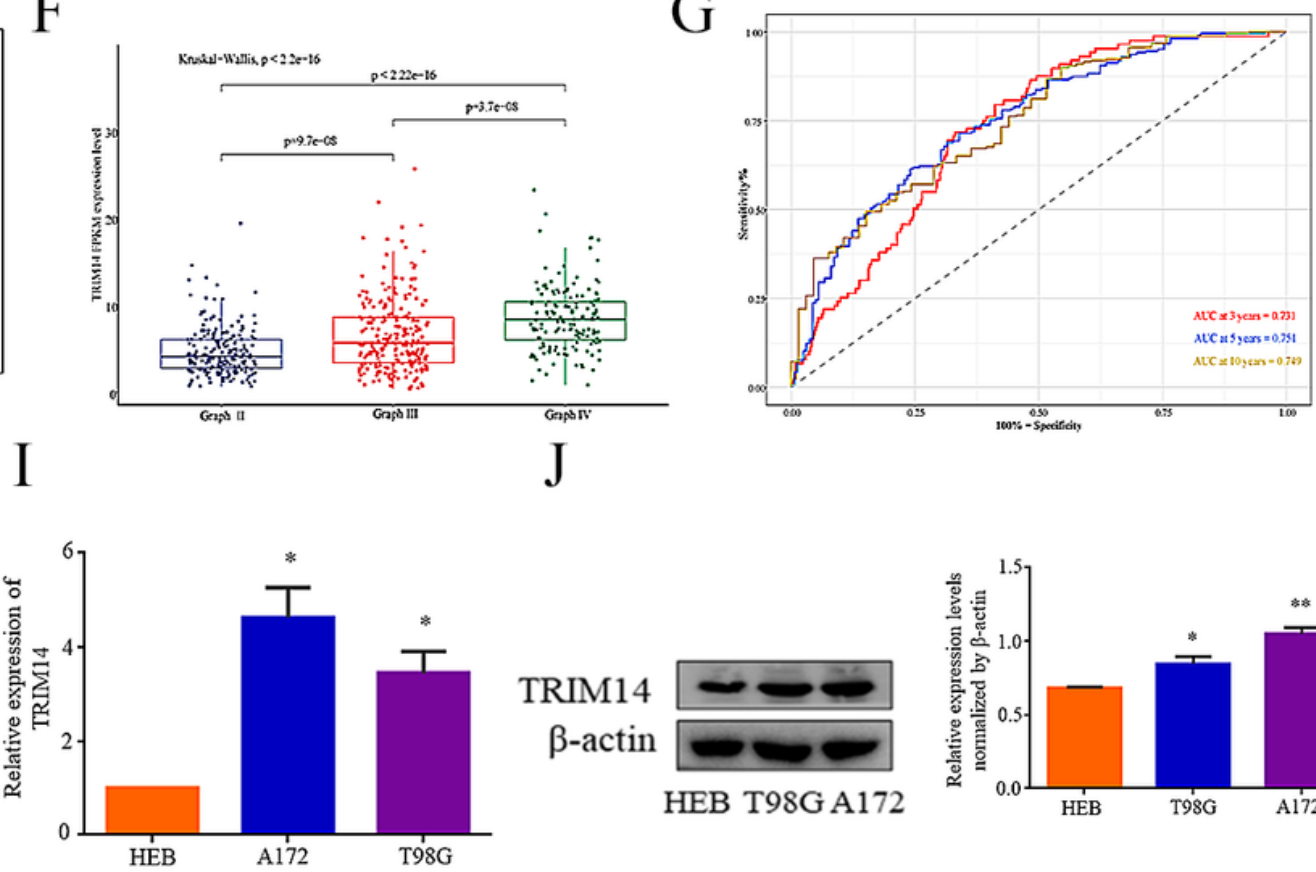

TRIM14

$\beta$-actin

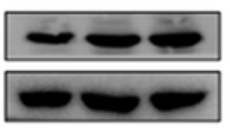

HEB T98G A172

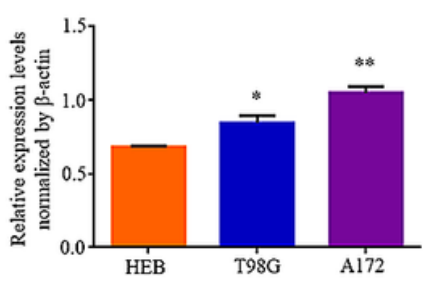

\section{Figure 5}

TRIM14 is a direct target of miR-6893-3p in glioma. (A) Heat map showing the differentially expressed genes mediated by LINC01578 knockdown. (B) The IncRNA-miRNA-mRNA ceRNA network based on the differentially expressed genes after LINC01578 knockdown and predicted target genes of miR-6893-3p. Square: IncRNAs; Triangle: miRNAs; Circle: mRNAs. (C) Take the intersection of the 5 miRNA target genes (miRanda, TargetScan, RNAhybrid bioinformatics databases were used to predict the target genes) and the list of differentially expressed genes in the LINC01578 knockdown cells analyzed by RNA sequencing 
in Figure 5A. (D) The predicted binding sites between miR-6893-3p and TRIM14. Dual luciferase reporter assay was used to determine the luciferase activity in 293T cells co-transfected with wild-type (WT) or mutant (MUT) TRIM14 and miR-6893-3p mimics or miR-6893-3p inhibitor. (E) The GEPIA2 database was used to analysis the TRIM14 expression in glioma tissues (GBM and LGG) and the relationship between low with high TRIM14. (F) The TCGA database was used to analysis the TRIM14 expression in the WHO grade of glioma patients. (G) ROC curve for predicting glioma based on LINC01578 expression. (H) RTqPCR was used to detect the expression of TRIM14 in glioma tissues. (I) RT-qPCR was used to detect the expression of TRIM14 in normal human brain cell line (HEB) and glioma cell lines (T98G, A172). (J) Western blot was used to detect the expression of TRIM14 in normal human brain cell line (HEB) and glioma cell lines (T98G, A172). Data are mean \pm SD from three independent experiments. ${ }^{*} P<0.05$, ** $P$ $<0.01$, *** $P<0.001$. 

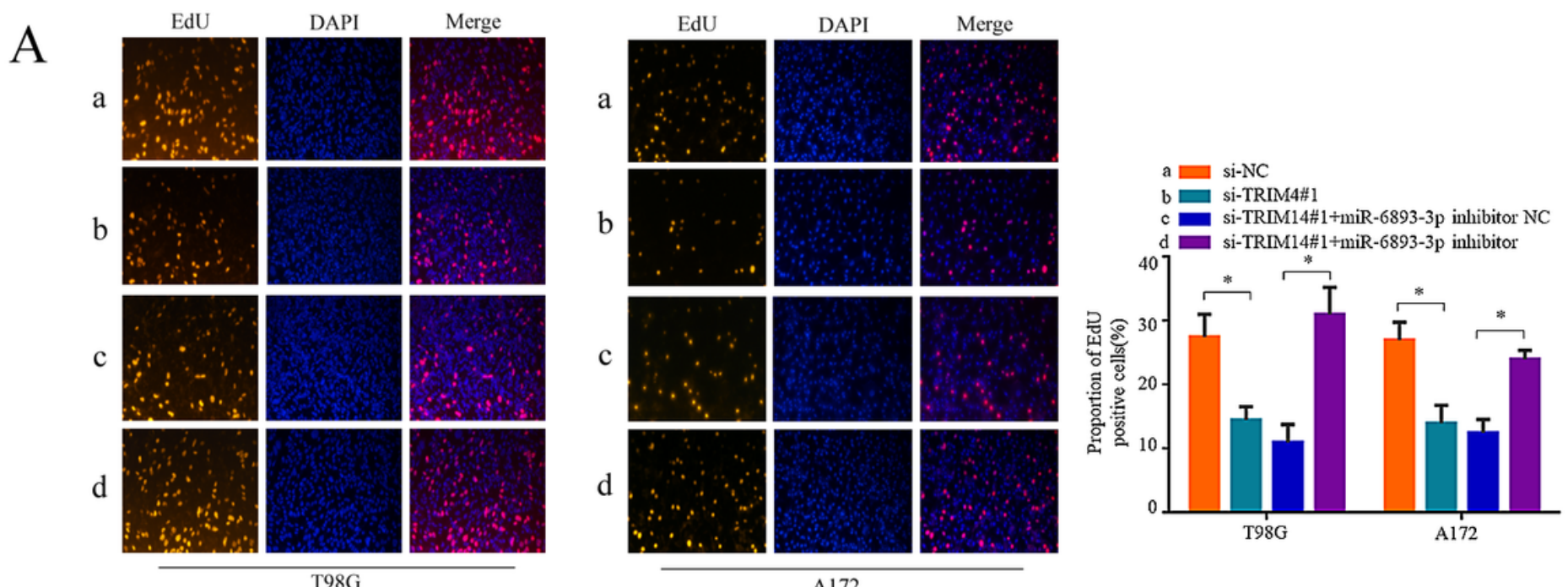

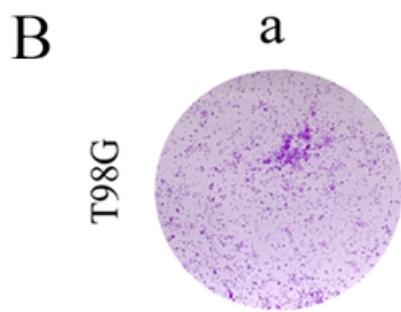

a

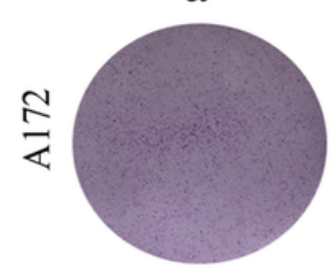

C

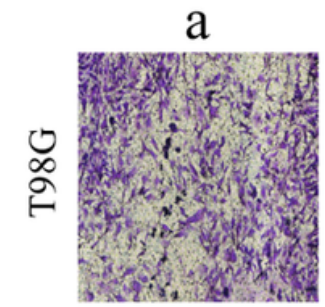

a b

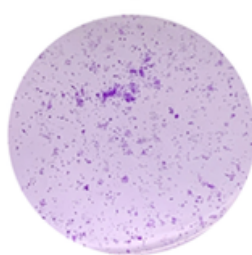

b

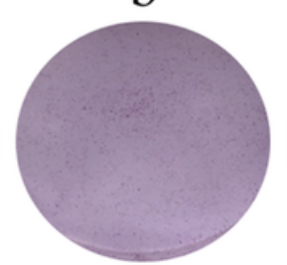

b

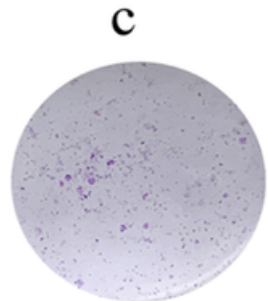

c

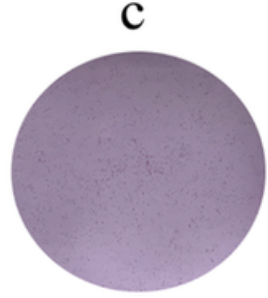

c
A172

d

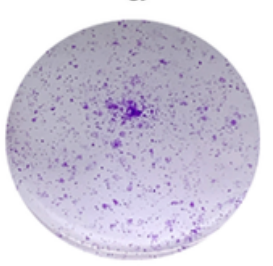

d

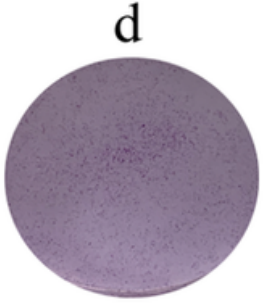

d

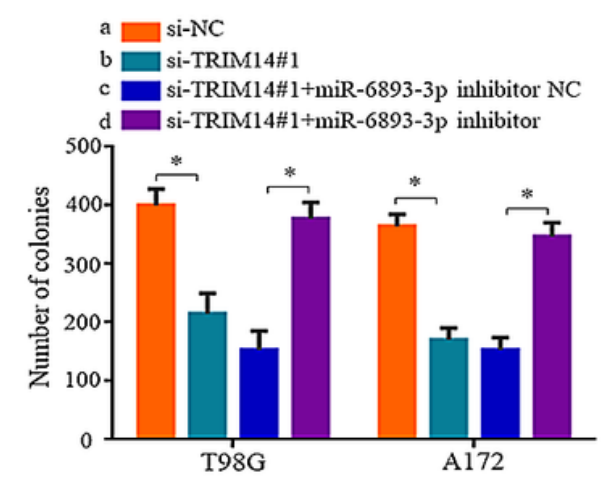

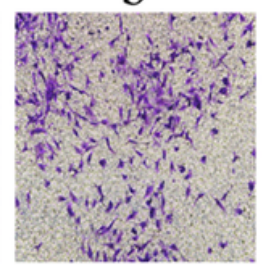

b

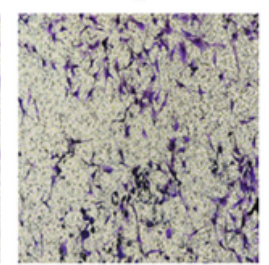

c

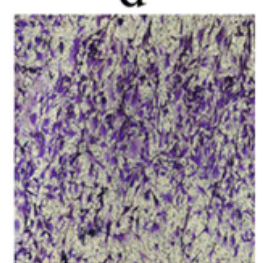

d
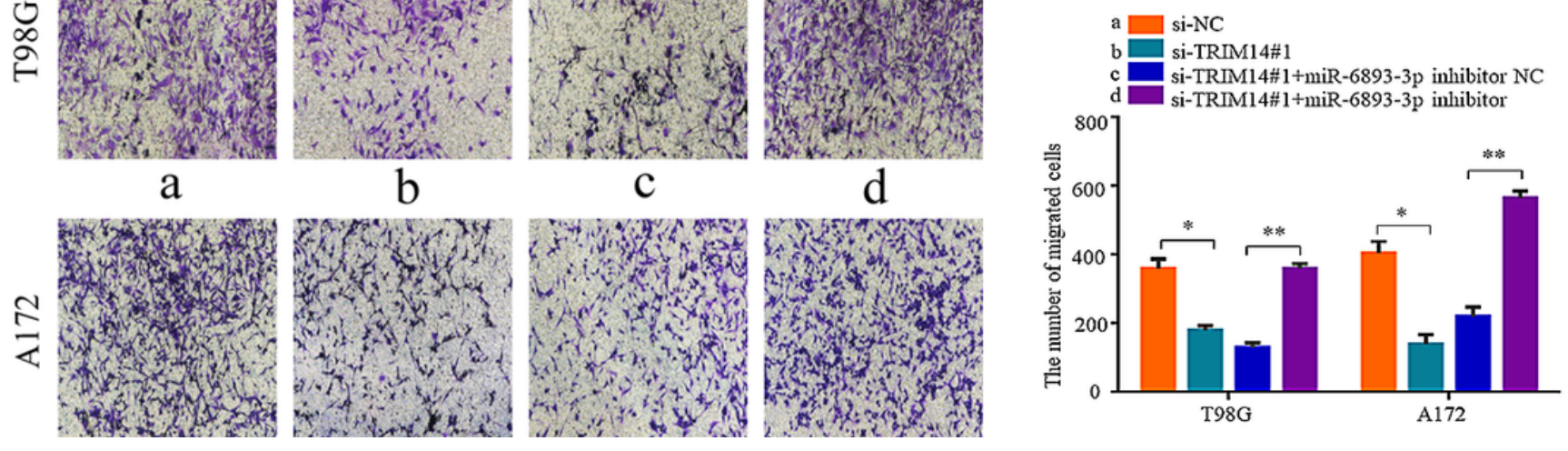

Figure 6

TRIM14 contributes to the function of miR-6893-3p. (A) EdU assay was employed to determine the proliferative ability of T98G and A172 cells co-transfected with siTRIM14 and/or miR-6893-3p inhibitor. Percentage of EdU positive cells was quantified using the GraphPad. (B) Colony formation in T98G and A172 cells co-transfected with siTRIM14 and/or miR-6893-3p inhibitor was performed. Clone numbers was quantified using the GraphPad. (C) The migration of T98G and A172 cells co-transfected with siTRIM14 and/or miR-6893-3p inhibitor was determined by transwell assays. The migration cells were 
quantified using the GraphPad. Data are mean \pm SD from three independent experiments. * $P<0.05, * \star P$ $<0.01, * \star \star P<0.001$.
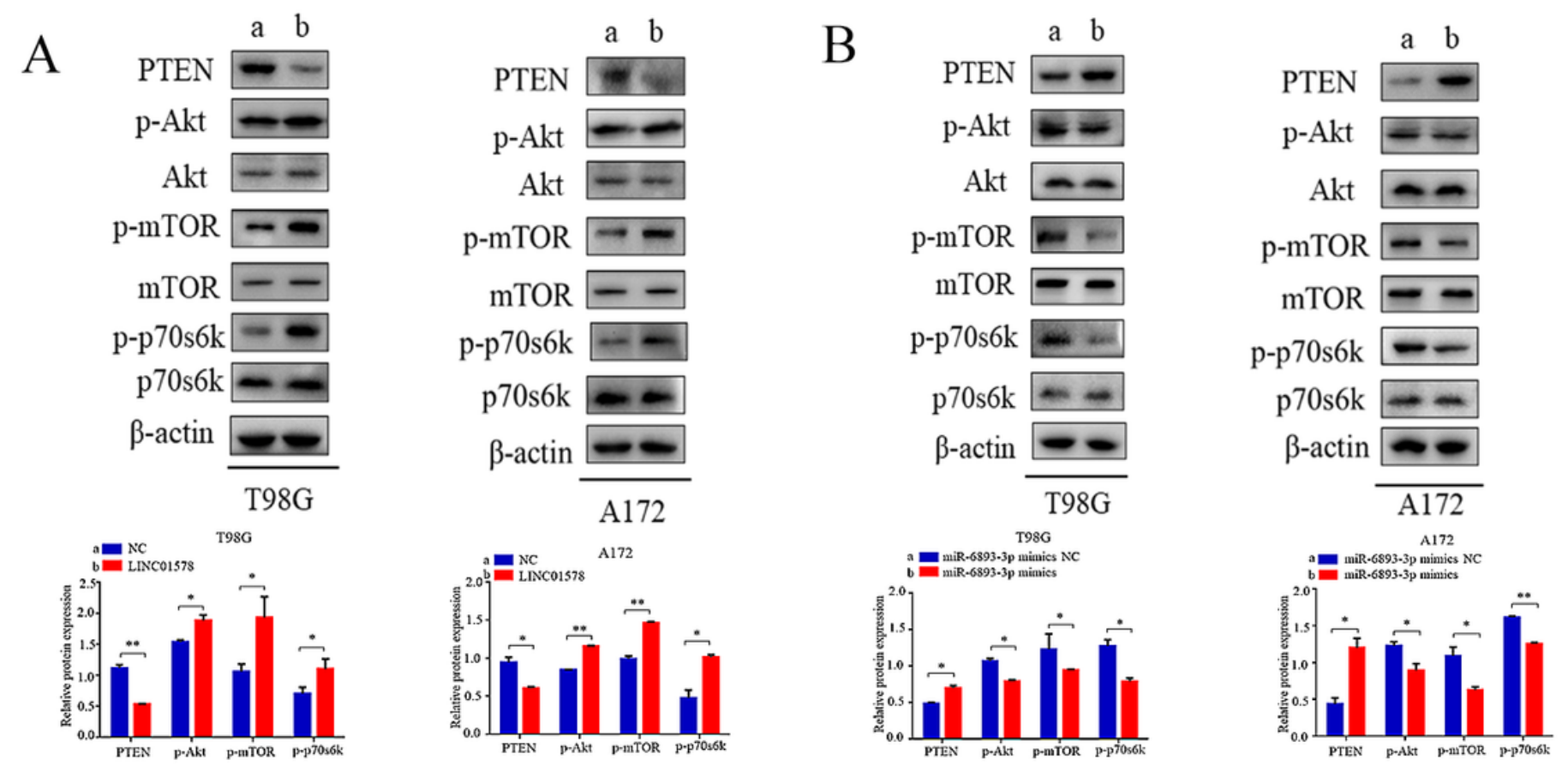

C

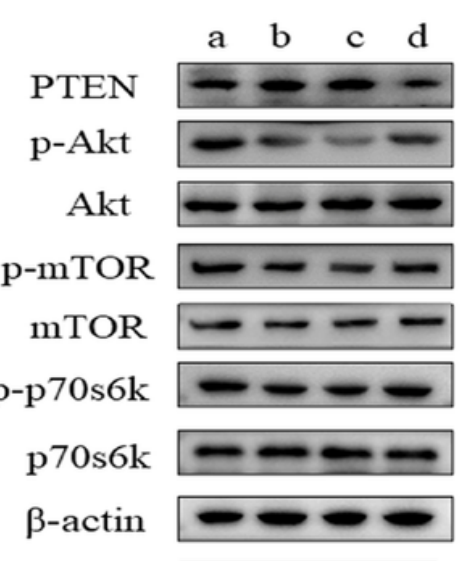

T98G

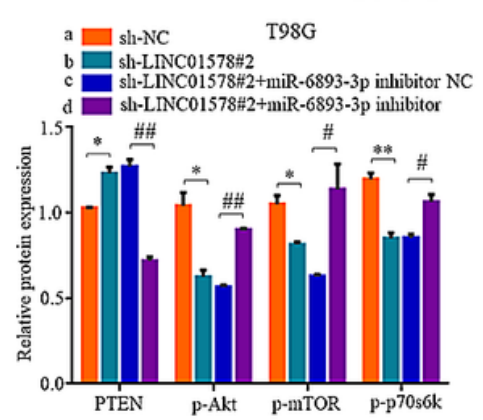

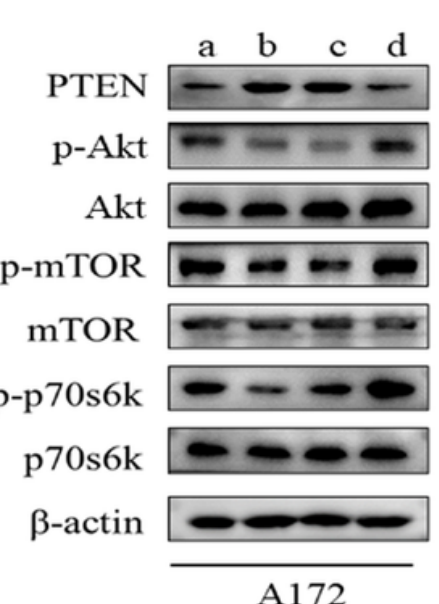

A172

A.172

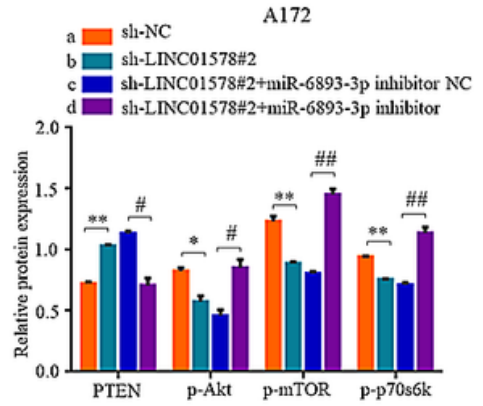

D

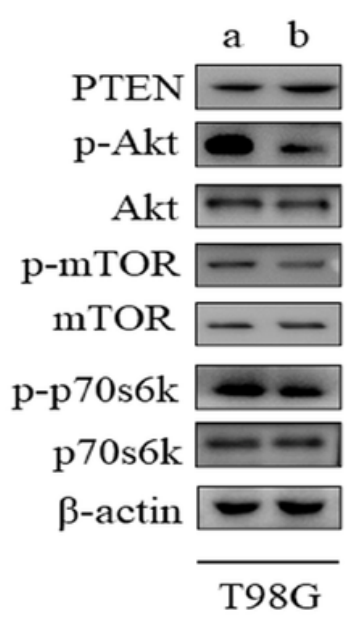

PTEN $-\frac{\mathrm{a} b}{-}-$

p-Akt

Akt

p-mTOR

mTOR

p-p70s6k

p70s6k

$\beta$-actin

A172
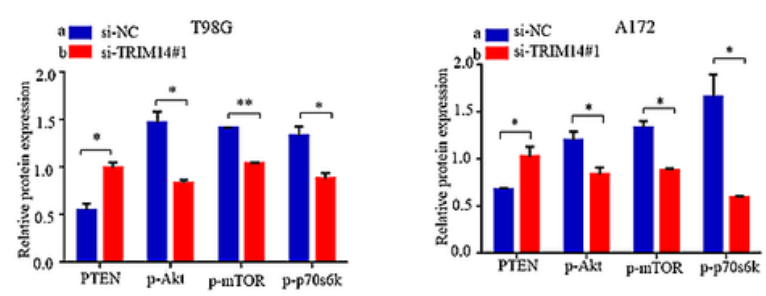

Figure 7

LINC01578 promote the Akt/mTOR/P70S6K pathway. (A) Western blot was used to detect the expression of indicated genes of T98G and A172 cells with LINC01578 overexpression. (B) Western blot was used to detect the expression of indicated genes of T98G and A172 cells with miR-6893-3p overexpression. 
Western blot was used to detect the expression of indicated genes of T98G and A172 cells co-transfected with shLINC01578 and/or miR-6893-3p inhibitor. (D) Western blot was used to detect the expression of indicated genes of T98G and A172 cells with TRIM14 knockdown. Data are mean \pm SD from three independent experiments. ${ }^{*} P<0.05, * \star P<0.01, * \star * P<0.001$.

A

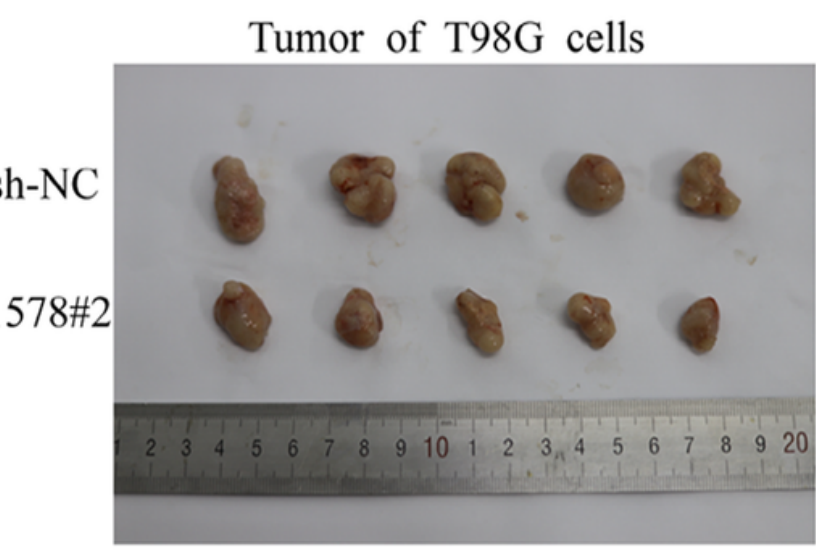

sh-LINC01578\#2
D

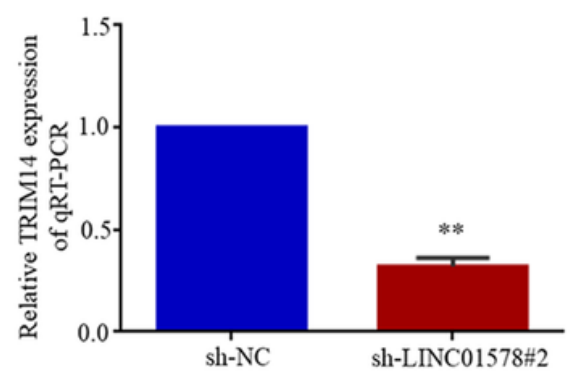

B

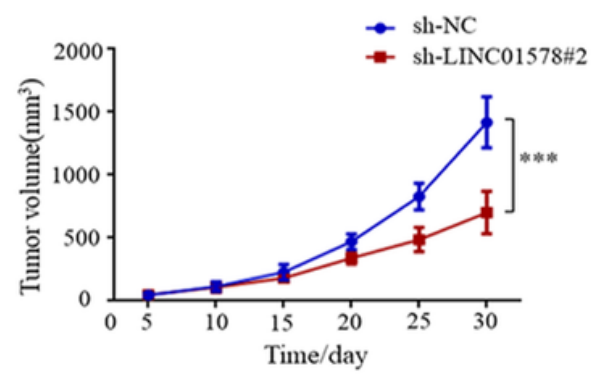

$\mathrm{C}$

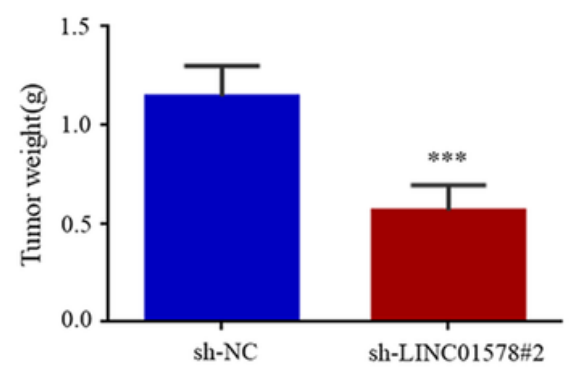

$\mathrm{E}$

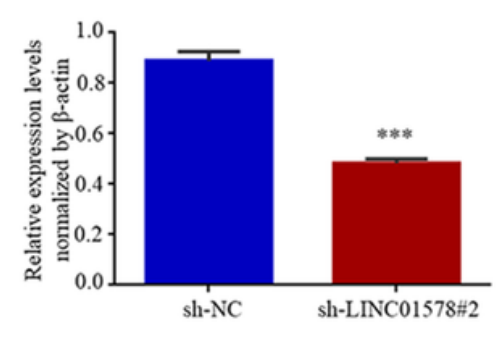

\section{Figure 8}

LINC01578 facilitates glioma growth in vivo. (A) A xenograft murine model was built using the T98G cells transfected with shLINC01578 or shNC. Tumors were collected from nude mice at 30 days. (B) The glioma volume curves were detected for 30 days. (C) Statistics of the weight of the tumors in A. (D) The TRIM14 mRNA expression in shLINC01578 and shNC groups was detected by RT-qPCR. (E) The TRIM14 protein expression in shLINC01578 and shNC groups was detected by western blot. Data are mean \pm SD from three independent experiments. ${ }^{*} P<0.05,{ }^{*} P<0.01$, $* \star \star ~ P<0.001$. 

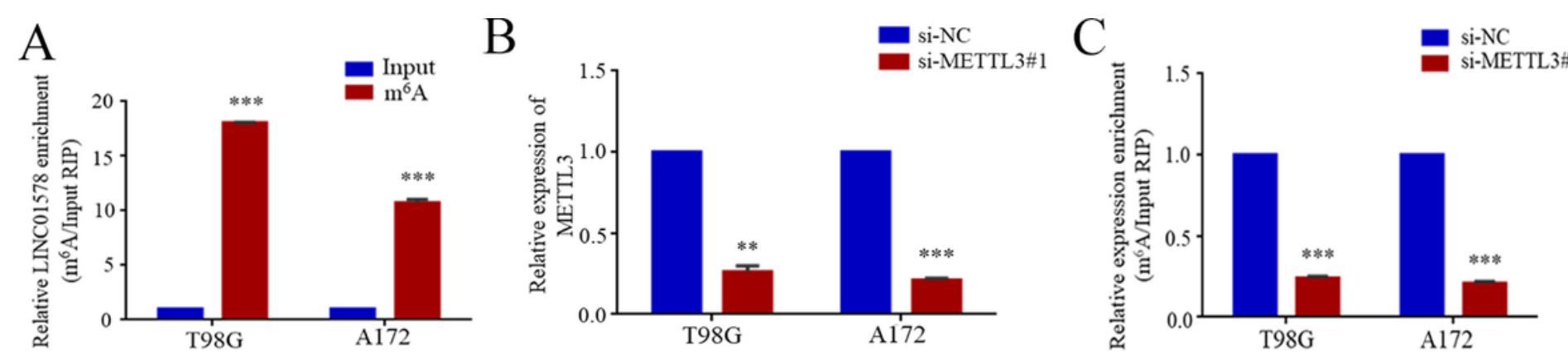

$\mathrm{D}$

$\mathrm{E}$

$\mathrm{F}$
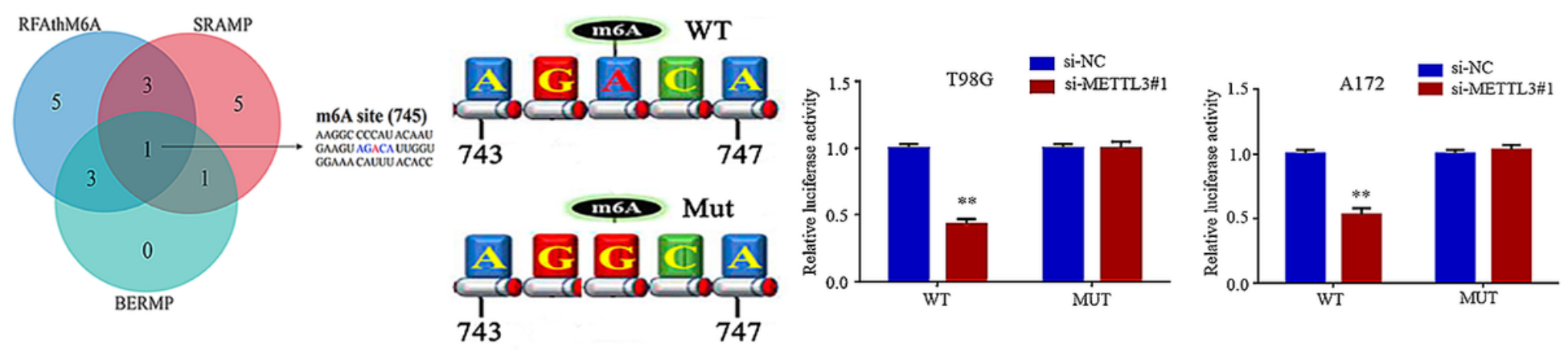

$\mathrm{G}$
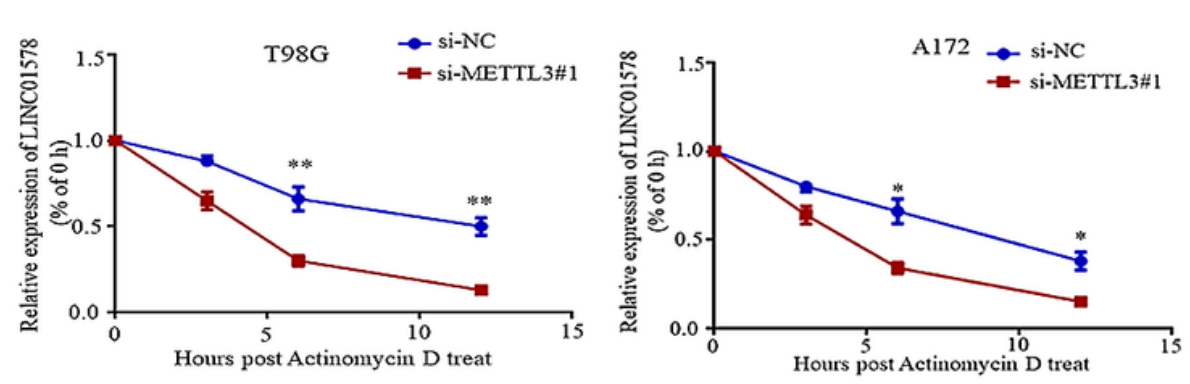

$\mathrm{H}$

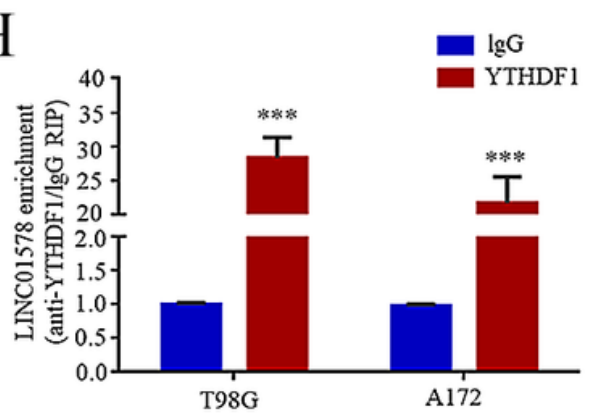

I
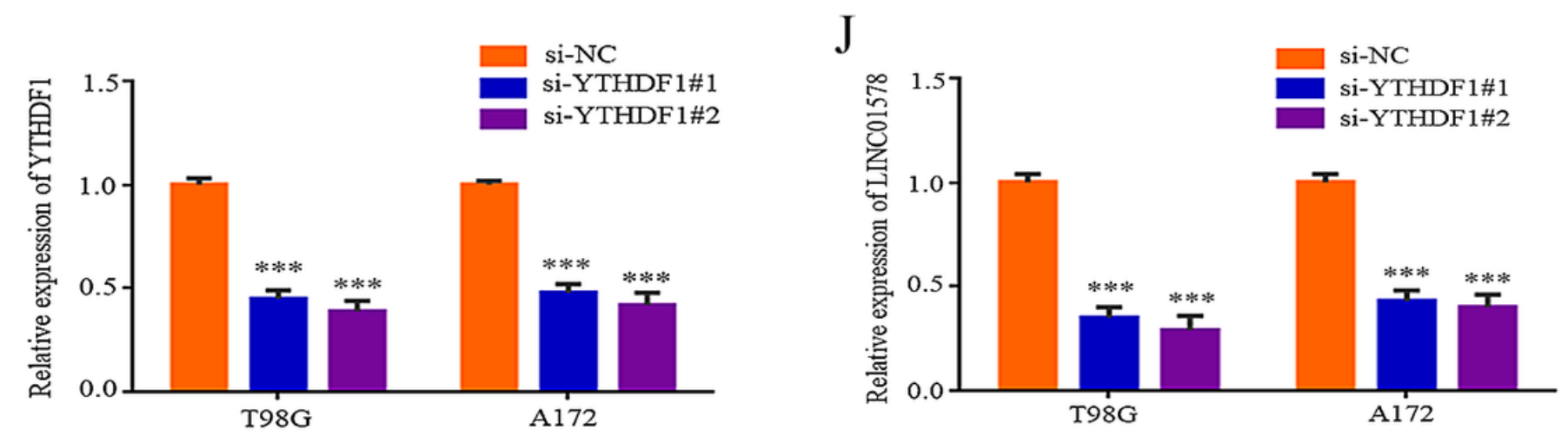

Figure 9

$\mathrm{m}^{6} \mathrm{~A}$ modification is involved in the upregulation of LINC01578 in glioma. (A) $\mathrm{m}^{6} \mathrm{~A}$ RIP-qPCR analysis of LINC01578 in T98G and A172 cells. (B) The LINC01578 expression was detected in T98G and A172 cells with METTL3 knockdown. (C) $\mathrm{m}^{6}$ A RIP-qPCR analysis of LINC01578 was detected in T98G and A172 cells with METTL3 knockdown. (D) $\mathrm{m}^{6} \mathrm{~A}$ modification sites of LINC01578 were predicted by RFAthM6A, SRAMP, BERMP databases. (E) Schematic representation of the wild-type or mutant $\mathrm{m}^{6} \mathrm{~A}$ motifs within LINC01578. (F) Mutation of $\mathrm{m}^{6} \mathrm{~A}$ consensus sequences or knockdown of METTL3 promoted the 
posttranscriptional repression of LINC01578 in T98G and A172 cells. (G) RT-qPCR was used to detect the expression of LINC01578 in T98G and A172 cells which transfected with siMETTL3 and treated with actinomycin D for the indicated times. (H) Anti-YTHDF1 RIP-qPCR analysis of LINC01578 in T98G and A172 cells. (I) RT-qPCR showed that the inhibitory efficiency of YTHDF1 in T98G and A172 cells. (J) RTqPCR was performed to detect the expression of YTHDF1 and LINC01578 in T98G and A172 cells with

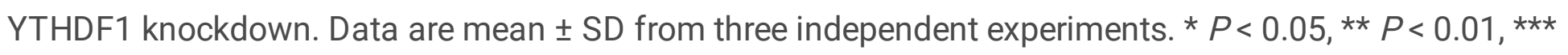
$P<0.001$.

\section{Supplementary Files}

This is a list of supplementary files associated with this preprint. Click to download.

- SupplementaryFiguresandFigureLegends.docx 